Graphical abstract

SAXS-scored structural models

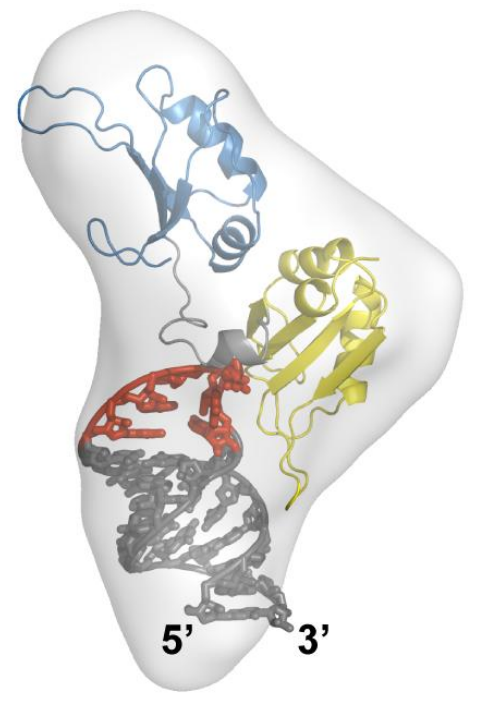

NSD $=0.8$

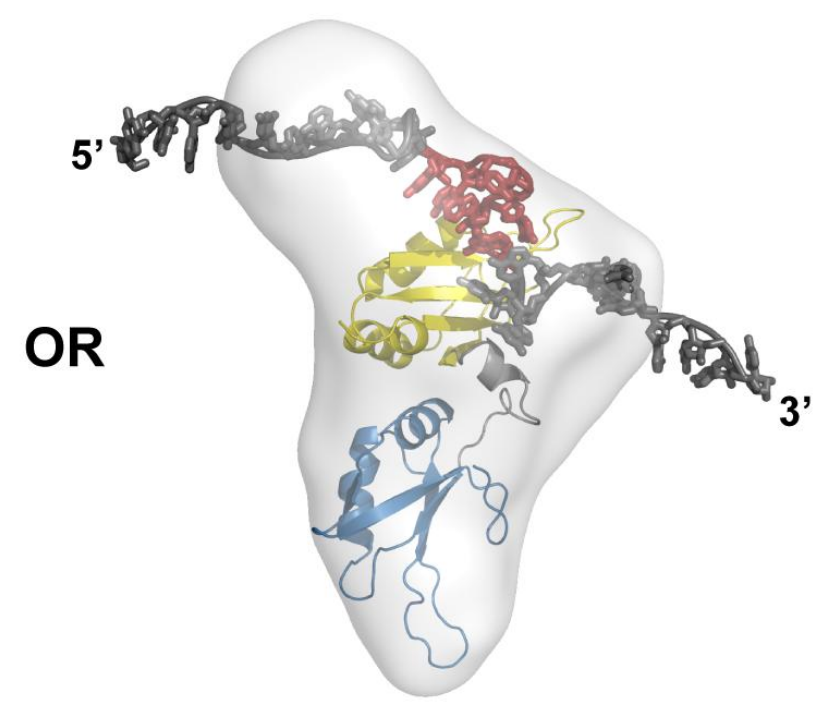

NSD $=1.0$ 


\title{
The First Crystal Structure of the UP1 Domain of hnRNP A1 bound to RNA Reveals a New Look for an Old RNA Binding Protein
}

\author{
Christopher E. Morgan ${ }^{\dagger \neq}$, Jennifer L. Meagher ${ }^{\dagger \xi}$ Jeffrey D. Levengood, James \\ Delproposto $^{\S}$, Carrie Rollins ${ }^{*}$, Jeanne A. Stuckey and Blanton S. Tolbert $^{* *}$ \\ Department of Chemistry, Case Western Reserve University, Cleveland, $\mathrm{OH}$ \\ ${ }^{\S}$ Life Sciences Institute, University of Michigan, Ann Arbor, MI \\ ${ }^{\dagger}$ Contributed equally to this work \\ *Corresponding author \\ Blanton S. Tolbert \\ Phone: 216-368-0605 \\ Email: bst18@case.edu
}




\section{Introduction}

Heterogeneous nuclear ribonucleoproteins (hnRNPs) of the A/B subfamily participate in a wide range of RNA biological events, including roles in RNA splicing, microRNA biogenesis, mechanisms of transcriptional control, mRNA trafficking and as trans factors to stimulate internal ribosomal entry ${ }^{1 ; 2}$. Related to these varied functions, hnRNP A/B members have a modular domain organization consisting of tandem N-terminal RNA Recognition Motifs (RRMs) followed by C-terminal auxiliary domains often enriched in glycine and small polar residues ${ }^{1 ; 2}$. The RRMs confer sequence specific nucleic acid binding properties, whereas the $\mathrm{C}$-terminal domains primarily mediate homologous and heterologous protein-protein interactions. Thus, specific hnRNP A/B interactions with their cognate RNA targets establish signaling networks that impact a broad array of cellular and viral processes.

Of the A/B members, the biological functions of hnRNP A1 have been the most thoroughly characterized. Human hnRNP A1 exists as two alternatively spliced isoforms, A1-A and A1-B. The A1-B isoform encodes the full-length protein of 372 amino acids. A1-A, the most abundant isoform, is an alternatively spliced variant that has 50 residues removed from its C-terminal domain. The structural organization of hnRNP A1 follows that of other A/B members, with tandem N-terminal RRMs (RRM1 and RRM2) and a glycine-rich C-terminal region (Figure 1A). Within the C-terminus, hnRNP A1 contains an RGG box purportedly involved in non-specific RNA binding along with an M9 sequence responsible for nucleo-cytoplasmic shuttling ${ }^{3}$. The tandem RRM domains, which are collectively known as UP1 (residues 1-196), display a high level of sequence and structural similarity ${ }^{4 ;}$. Both RRMs adopt the consensus $\beta 1 \alpha 1 \beta 2 \beta 3 \alpha 2 \beta 4$ fold where $\beta 1$ and $\beta 3$ comprise the conserved RNP2 and RNP1 nucleic acid interacting motifs, respectively. Despite the overall similarities between the RRM domains, they appear to have evolved distinct alternative splicing functions ${ }^{6}$. Domain swap, deletion and duplication studies suggest the RRMs are indeed non-redundant and functionally nonequivalent ${ }^{6}$.

Structural insights into the RNA processing functions of hnRNP A1 are derived from structures of its UP1 domain in an unbound state and bound to single strand DNA ${ }^{4 ; 5 ; 7}$. In all the DNA bound structures, the complex forms a dimer about a crystallographic 2-fold axis where two copies of a 12-nt ssDNA fragment transverse the dimer interface (Figure S1). The 5' half of either DNA strand interacts with the beta sheet surface of RRM1, whereas the 3' half stretches across the dimer interface to interact with RRM2. In these structures, the DNA strands run in an antiparallel direction where identical contacts are observed between the RRM domains and repeated copies of a 5'-TAG-3' sequence element (Figure S1). Since the spatial orientation of RRM1 and RRM2 are also antiparallel, the TAG elements maintain a 5'-3' polarity relative to the beta sheet surface as the chain transverses the dimer interface ${ }^{7}$. The UP1-DNA structures have provided valuable insights into features of sequence specific nucleic acid recognition; however, they do not explain how hnRNP A1 interacts with its RNA targets that do not typically exist as linear strands but instead adopt complex secondary and tertiary structures $8 ; 9 ; 10 ; 11$; $12 ; 13$ 
RNA stem loops represent a major class of natural hnRNP A1 targets, yet little is known about the mode of recognition. Indeed, hnRNP A1 binds stable RNA stem loop structures to mediate cap independent translation ${ }^{14 ; 15 ; 16}$, microRNA biogenesis ${ }^{9 ; 10 ; 11 ; 12 ; 13}$ and alternative splice site selection $^{8 ; 17 ; 18 ; 19}$. RNA chemical protection studies of hnRNP A1 bound to pri-miRNA-18a and HIV splicing silencers show discrete protection patterns, unlike the binding interface observed in UP1-DNA crystal structures ${ }^{17 ; 19 ; 20}$. Thus, it is unclear as to how hnRNP A1 interacts with stable RNA stem loop structures, where only a few bases are exposed for recognition.

In an attempt to determine the X-ray crystal structure of UP1 bound to the HIV Exon Splicing Silencer 3 stem loop (SL3 ${ }^{\mathrm{ESS} 3}$ ), we obtained high quality crystals of UP1 bound to the three nucleotide loop recognition element, 5'-AGU-3'. Structure determination to $1.92 \AA$ resolution reveals that the RRM1 and inter-RRM linker fold to form a "nucleobase pocket" that sequesters the RNA. RRM2 does not interact with the RNA. To further investigate the structural basis of UP1-SL3 ${ }^{\mathrm{ESS} 3}$ recognition, we used SAXS scattering profiles to filter data-driven structural models of the complex. The model reveals UP1 binds the apical loop of SL3 ${ }^{\text {ESS3 }}$ through its RRM1 domain and inter-RRM linker only. We further probed the binding interface by carrying out a series of kinetic studies with SL3 ${ }^{\mathrm{ESS} 3}$ mutants. Mutations within the apical loop at AG dinucleotide steps affect the binding kinetics by slowing down the rate of complex formation. Structureguided mutagenesis of conserved salt-bridge interactions located opposite the RNA binding surface reveals RRM1 and RRM2 are coupled, which positions the inter-RRM linker in a conformation for high-affinity and specific binding along the RRM1 surface. Taken together, this study provides structural insights into the mechanisms hnRNP A1 uses to recognize RNA stem loop targets, which has significant implications for its diverse roles in cellular and viral RNA processing events.

\section{Results}

Our previous biophysical studies revealed both RRM domains of UP1 are required to bind HIV SL3 ${ }^{\text {ESS3 }}$ with nanomolar affinity ${ }^{21 ; 22}$, where the recognition surface of SL3 ${ }^{\text {ESS3 }}$ was localized to the GAUUAG apical loop (Figure 1B). In agreement with those earlier studies, ${ }^{1} \mathrm{H}-{ }^{13} \mathrm{C}$ HMQC titrations of UP1 into a ${ }^{13} \mathrm{C}(\mathrm{A})$-labeled SL3 ${ }^{\mathrm{ESS} 3}$ construct showed a significant chemical shift perturbation (CSP) of A8458 H2, with only minor CSPs observed at other labeled sites (Figure 1C). These results agree well with our published saturation transfer difference NMR spectra where specific transfer was observed to hydrogen atoms attached to A8458 and neighboring nucleotides ${ }^{22}$. Taken together, the results demonstrate both RRM domains are needed to bind SL3 ${ }^{\mathrm{ESS} 3}$ site-specifically with high affinity, and without inducing global unfolding of the RNA structure.

Structure of the UP1-(AGU) complex reveals RNA binding through the RRM1 surface and inter-RRM linker only

To better understand how UP1 recognizes SL3 ${ }^{\text {ESS3 }}$, crystal screens of a 1:1 UP1-SL3 ${ }^{\text {ESS3 }}$ complex were pursued. Instead of the intact complex, we obtained crystals of UP1 bound 
to a short 5'-AGU-3' trinucleotide motif (Figure 2A). The structure was solved by molecular replacement using the unbound UP1 structure (pdb code 1L3K) as the search model. When the structure was solved, there was one molecule of UP1 with two RRM domains exhibiting the conserved $\beta 1 \alpha 1 \beta 2 \beta 3 \alpha 2 \beta 4$ fold in the asymmetric unit. The initial difference $(\mathrm{Fo}-\mathrm{Fc})$ electron density map revealed the presence of RNA bound to the RRM1 domain and inter-RRM linker. At $1.92 \AA$ resolution, we were able to confidently model the 5'-AGU-3' motif into the electron density (Figure 2B), even though the SL3 ${ }^{\text {ESS3 }}$ primary sequence contains three RRY motifs (GAU, AGU and AAC). The (FoFc) omit map clearly shows an $\mathrm{rA}$ in position 1 and $\mathrm{rG}$ in position 2 (Figure S2), making AGU the only possible RRY motif that fits the electron density. Presumably, SL $3{ }^{\text {ESS } 3}$ was partially hydrolyzed during crystal growth, where the 5'-AGU-3' motif was protected as a result of direct contact with UP1. As shown in figure 1A, the 5'-AGU-3' sequence derives from the apical loop of SL $3^{\mathrm{ESS} 3}$. The primary binding epitope observed in the crystal agrees with our observed ${ }^{1} \mathrm{H}-{ }^{13} \mathrm{C}$ HMQC titrations (Figure 1C) and previous binding studies, where we mapped the interaction surface using NMR and mutagenesis ${ }^{21}$; 22

The beta sheet surface of RRM1 and the inter-RRM linker (residues 90-105) together form a "nucleobase pocket" to enclose the rA and rG residues, which both adopt a syn conformation about the glycosidic bond. The uracil base is anti and is positioned outside the pocket (Figure 2A). The inter-RRM linker is ordered into a $3_{10}$ helix as seen in the DNA bound structure due to extensive intermolecular interactions with both nucleobases. The stereochemical and electrostatic features of the nucleobase pocket result in several stabilizing interactions with the RNA (Figure 2C and 2D). His101 and Phe17 $\pi-\pi$ stack above and below the aromatic ring of rA, respectively. In this orientation, the rA base makes 2 hydrogen bonds with the protein backbone. One occurs between N7 along the Hoogsteen edge and the backbone amide of Val90 from the inter-RRM linker. The second hydrogen bond is between N6 and the backbone carbonyl of Arg88. The $\gamma-$ hydroxyl group of Ser95 from the inter-RRM linker is within hydrogen bond distance of the rA 2' hydroxyl group. Additional interactions stabilizing rA include hydrophobic packing of Phe57 against the ribose sugar.

At the second nucleobase position, the guanidinium group of Arg92 forms a cation- $\pi$ interaction with the 5-membered ring of $\mathrm{rG}$ (Figure 2C). The guanine base is further stabilized through $\pi-\pi$ stacking interactions with Phe59. The carbonyl oxygen of $\mathrm{rG}$ interacts with the $\varepsilon$-amino group of Gln12 and the $\zeta$-amino group of Lys 15 donates a hydrogen bond to the N7 position along the Hoogsteen edge. Additionally, the guanidinum group of Arg92 donates a hydrogen bond to the 2'hydroxyl and the backbone carbonyl of Val90 is within hydrogen bonding distance of N1 and N2.

\section{Comparison to the interactions observed in UP1-DNA structure}

The intermolecular contacts between RRM1 and the rA and rG nucleotides in the RNA bound structure are similar to the binding interactions between RRM1 and the dA3 and dG4 nucleotides in the DNA bound structure (Figure S3A) with the exception of the orientation of the adenine base. The base of $\mathrm{dA} 3$ is in the anti conformation, while the $\mathrm{rA}$ 
base conformation is syn. This change in conformation is due to a hydrogen bond between the 2'O of the ribose ring of rA and Ser95 (Figure 2C). This interaction shifts the position of the ribose causing the base to flip conformations. Due to the arrangement of the nitrogens (N6 and N7) on the adenine base, the hydrogen bonding of the base to UP1 is similar between the DNA and RNA bound structures. The direct hydrogen bond between the $\mathrm{N} 3$ atom of adenine and the $\mathrm{N} 2$ of guanine that is present in the DNA bound structure is lost in the RNA bound structure. To compensate for this loss, a water molecule mediates the interaction of $\mathrm{O} 2$ ' atom of $\mathrm{rA}$ with $\mathrm{N} 2$ of $\mathrm{rG}$ through hydrogen bonding. Other differences between the DNA and RNA bound structures occur with the 3' ends. dG5 is bound to the RRM1 domain with its base interacting with Arg92 (Figure $\mathrm{S} 2 \mathrm{~A})$. The $\mathrm{rU}$ base pivots away from the RRM1 domain to interact with symmetry related UP1 in the crystal interface (Figure S3B). The OP1 atom of the rU phosphate group hydrogen bonds with the NH1 and NH2 atoms of Arg55 (Figure 2C).

\section{Structural Model of UPI-SL3 ${ }^{E S S 3}$ Complex}

To gain structural insights into how UP1 recognizes intact SL3 ${ }^{\mathrm{ESS} 3}$, we determined a data-driven (HADDOCK) ${ }^{23 ; 24}$ structural model of the complex. A detailed description of the approach is provided in materials and methods but in brief, we followed a protocol similar to the one described by Butcher et $a l^{25}$, with the exception that our HADDOCK clusters were scored against small angle $x$-ray scattering (SAXS) profiles ${ }^{26}$ and the final model was not further refined through simulation. Selection of experimental data to guide the docking routine included the following ambiguous interaction restraints: (i) proteinRNA interactions observed in the UP1-(AGU) crystal structure; (ii) interactions detected from saturation transfer difference $\mathrm{NMR}^{22}$; (iii) ${ }^{1} \mathrm{H}_{-}{ }^{13} \mathrm{C}$ CSPs derived from titrations of ${ }^{13}$ C-labeled SL3 ${ }^{\text {ESS3 }}$ constructs with unlabeled UP1 (Figure 1C); and (iv) thermodynamic perturbations observed from calorimetric titrations of SL3 ${ }^{\mathrm{ESS} 3}$ apical loop mutants with $\mathrm{UP}^{22} \cdot{ }^{1} \mathrm{H}-{ }^{15} \mathrm{~N}$ CSPs of UP1 following addition of unlabeled SL3 ${ }^{\mathrm{ESS} 3}$ were not used due to complications of differentiating between direct interactions and binding induced conformational changes ${ }^{22}$.

A representative SEC-SAXS scattering profile collected on an $\sim 1.2: 1$ UP1:SL3 ${ }^{\text {ESS3 }}$ complex is shown in figure $3 \mathrm{~A}$. Analysis of the Guinier plot of the complex revealed an $\mathrm{R}_{\mathrm{g}}$ of $25.02 \pm 2.81 \AA$ and a MW estimate equal to $30.7 \mathrm{kDa}$, which is in agreement with the expected MW (33.8 kDa) of a 1:1 complex. The $\rho(\mathrm{r})$ pair distribution function (Figure $3 \mathrm{~B})$ gave an $\mathrm{R}_{\mathrm{g}}$ of $\sim 26 \AA$, consistent with the Guinier analysis and a maximum dimension $\left(\mathrm{d}_{\max }\right)$ of $93.5 \AA$. Ab initio reconstructions were determined from the $\rho(\mathrm{r})$ function using DAMMIF/DAMAVER ${ }^{27 ; 28}$ and provide a normalized spatial discrepancy (NSD) value of $0.61 \pm 0.02$. The reconstruction reveals an overall chevron-like shape for the intact UP1SL3 ${ }^{\mathrm{ESS} 3}$ complex (Figure 3C).

Thirteen HADDOCK clusters were filtered against the experimental SAXS data by back calculating scattering profiles using CRYSOL ${ }^{29}$ and then ranked according to their $\chi^{2}$ values (Table S1). The average $\chi^{2}$ values of the best and worst clusters are $0.99 \pm 0.02$ and $1.85 \pm 0.17$, respectively. Clusters with $\chi^{2}$ values greater than 1.3 were removed from further analysis. The five lowest energy structures from the remaining clusters were 
further filtered based on their NSD values to the SAXS reconstructions calculated using SUPCOMB $^{30}$. The average NSD of the best scoring cluster is $0.80 \pm 0.01$ indicating very good agreement between the structural models and SAXS reconstruction. A representative SAXS-filtered structural model of the UP1-SL3 ${ }^{\text {ESS3 }}$ complex fit into the molecular reconstruction is shown in figure 4A. UP1 docks on the apical loop of SL3 ${ }^{\text {ESS3 }}$ using its RRM1 domain and inter-RRM linker only (Figure 4B). The center of the beta sheet surface of RRM2 is more than $20 \AA$ away from the apical loop. The model also reveals the $\beta 2 / 3$ loop of RRM1 is positioned to interact non-specifically with the phosphodiester backbone of the helical region. Indeed, the $\beta 2 / 3$ loop contains four conserved Arg and Lys residues. Loss of interactions between the $\beta 2 / 3$ loop and the phosphodiester backbone may partly explain the $\sim 150$ fold reduction in binding affinity of UP1 for a 6-nt ESS3 construct, where the lower helix of SL3 ${ }^{\text {ESS3 }}$ was deleted ${ }^{22}$.

Even though the SAXS results are consistent with a single UP1 molecule binding to a single SL3 ${ }^{\mathrm{ES} 3}$, it's conceivable the complex forms within the context of an unfolded RNA element, similar to the UP1-DNA complex (Figure S1). We tested the possibility by recalculating HADDOCK clusters where the ESS3 element was modeled in an extended conformation. SUPCOMB docking of a representative low-energy HADDOCK model gave an NSD value of $\sim 1.0$ indicating poor agreement with the molecular reconstruction (Figure S4). This result is consistent with our ${ }^{1} \mathrm{H}_{-}{ }^{13} \mathrm{C}$ HMQC titration shown in Figure $1 \mathrm{C}$, where correlation peaks derived from adenosines from the base paired region show very small CSPs, indicating the RNA stays folded within the complex. Collectively, these results indicate UP1 binds a folded SL3 ${ }^{\mathrm{ESS} 3}$ element as a 1:1 complex and in a manner where only the RRM1 domain and inter-RRM linker directly contact the RNA.

Kinetic binding studies of UP1 with $\mathrm{SL} 3^{\mathrm{ESS} 3}$ reveal cytosine substitutions affect rates of complex formation

The structural model reveals UP1 binds SL3 ${ }^{\mathrm{ESS} 3}$ using a mode of recognition - defined here by the oligomeric state, RNA conformation and stoichiometry - fundamentally distinct from the UP1-DNA structure (Figure 4B and S1). As described above, the UP1(AGU) crystal structure reveals detailed protein-RNA interactions; however, the interfacial contacts may be different within the context of an intact UP1-SL $3^{\text {ESS3 }}$ complex. To test the contribution of the SL3 ${ }^{\mathrm{ESS} 3}$ apical loop residues towards binding kinetics, we performed biolayer interferometry (BLI) studies on six SL3 ${ }^{\text {ESS3 }}$ mutants where each nucleotide of the 5'-GAUUAG-3' motif was changed to cytidine (Figure 5A and Figure S5). We previously showed R-to-C substitutions (where R = A or G) within this motif weaken the thermodynamic stability of the complex ${ }^{22}$. Here, we sought to determine whether the binding defects manifest as perturbations to the association or dissociation rates. BLI data shows that cytidine substitutions within the SL3 ${ }^{\mathrm{ESS} 3}$ apical loop affect binding affinities in a manner consistent with those determined by titration calorimetry (Figure 5B). Cytidine substitutions weakened the affinity of the UP1-SL3 ${ }^{\text {ESS3 }}$ interaction for every mutant studied except U8456C, which retained a similar $\mathrm{K}_{\mathrm{D}}$ value to that of wild type SL3 ${ }^{\mathrm{ESS} 3}\left(\log _{10}\right.$ Mutant/WT $\left.=-0.12\right)$. The largest increase in $\mathrm{K}_{\mathrm{D}}$ was observed for the 5'-GAUU $\underline{C G}-3$ ' construct $\left(\log _{10}\right.$ Mutant/WT $\left.=9.6\right)$, where the A-to-C 
substitution corresponds to the portion of the loop bound within the nucleobase pocket of UP1. The $\sim 10$ fold reduction in binding affinity observed for the 5'-GAUUCG-3' construct also agrees with our ${ }^{1} \mathrm{H}^{13}{ }^{13} \mathrm{C}$ HMQC titrations titrations, where A8458 H2 showed a significant downfield chemical shift perturbation (Figure 1C). Consistent with earlier observations, cytidine substitutions at other purine positions within the 5'GAUUAG-3' apical loop also weaken complex stability, albeit to a lesser degree (Table 2).

Interestingly, kinetic analysis showed slower association rates $\left(\mathrm{k}_{\mathrm{on}}\right)$ were the predominant mechanism that contributes to the changes in $\mathrm{K}_{\mathrm{D}}$, while the dissociation rates $\left(\mathrm{k}_{\text {off }}\right)$ remained largely unperturbed (Figure 5 and Table 2). Consistent with the higher $\mathrm{K}_{\mathrm{D}}$ value, the 5'-GAUUCG-3' construct shows the greatest reduction in $\mathrm{k}_{\text {on }}$ with an $\sim 10$ fold decrease relative to wild type. The observation that slower association rates account for the reductions in thermodynamic stability suggests the mutations fail to induce a proper conformational change in UP1 due to impaired binding within the nucleobase pocket; however, structural perturbations induced by the substitutions may also affect the local RNA conformation ${ }^{22}$. Thus, we conclude UP1 recognition requires proper structural and sequence features in order to form a stable complex with SL3 ${ }^{\mathrm{ESS} 3}$.

\section{Impact of UP1 salt-bridge mutations on RNA binding affinity}

Given the observation that RNA is only seen in the RRM1 binding site, we reasoned the binding preference of RRM1 over RRM2 results from the position of the inter-RRM linker. Indeed, the structure reveals the linker is positioned more towards the beta sheet surface of RRM1, where His101 and Arg92 extend out over the rA and rG bases, respectively. Since the relative orientations between the RRM domains are antiparallel, the position of the inter-RRM linker establishes a unique stereochemical environment along the beta sheet surface of RRM1 (Figure 2C and 2D). The fixed relative orientation of RRM1, RRM2 and the inter-RRM linker was also observed in the solution NMR structure of the unbound protein ${ }^{31}$. Two salt bridge interactions, R75:D155 and R88:D157 on the alpha helical side of the RNA binding surface (Figure 6A), are thought to stabilize the relative orientations of RRM1 and RRM2; however, the contributions of these interactions to the RNA binding properties of UP1 have not been determined ${ }^{4 ; 5 ; 7}$. To that end, we prepared a R75D/R88D UP1 double mutant (UP1 ${ }^{\mathrm{dm}}$ ), which was designed to decouple the RRM1-RRM2 interaction via electrostatic repulsions. Calorimetric titrations revealed UP1 ${ }^{\mathrm{dm}}$ binds $\mathrm{SL} 3^{\mathrm{ESS} 3}$ approximately 18 -fold weaker relative to the wild type protein (Figure S6). As shown in figure 6B, comparison of the thermodynamic signature reported here for UP1 ${ }^{\mathrm{dm}}$ with previously published values for the wild type protein clearly indicate a significant change in the nature of the intermolecular forces driving recognition $(\Delta \Delta \mathrm{H}=+29.8 \mathrm{kcal} / \mathrm{mol}$ and $\mathrm{T} \Delta \Delta \mathrm{S}=-28.2$ $\mathrm{kcal} / \mathrm{mol}$ ). The wild type protein forms more favorable intermolecular interactions with $\mathrm{SL}^{\mathrm{ESS} 3}$ than does UP1 ${ }^{\mathrm{dm}}$; however, the greater total binding enthalpy is offset by a greater total entropic penalty to binding. The differential thermodynamic signatures likely reflect conformational perturbations between $\mathrm{UP}^{\mathrm{dm}}$ and wild type UP1 protein, where the latter is in a conformation primed to bind RNA with high affinity (see below). 
To gain molecular insight into the influence of the salt-bridge mutations on UP1 structure, we performed all-atom MD simulations in explicit solvent using the ff12SB AMBER force field (see materials and methods). The results of the simulations for the double mutant and wild type UP1 are shown in figure 6C and trajectories are shown in figure S7. Analysis of the post-simulated structures reveal the nucleobase pocket of $\mathrm{UP} 1{ }^{\mathrm{dm}}$ undergoes large structural transitions relative to the wild type protein. As a metric, we measured the average $\mathrm{C} \alpha-\mathrm{C} \alpha$ distances of His101 and Arg92 to Phe residues 17, 57 and 59. At $0 \mathrm{~ns}$, the average distance between the $\mathrm{C} \alpha$ positions of His 101-Phe residues and Arg92-Phe residues is $10.2 \AA$ and $14.8 \AA$, respectively. Following $10 \mathrm{~ns}$ of simulation, these distances increase to $10.9 \AA$ and $16.9 \AA$, indicating the inter-RRM linker in the wild type protein experiences relatively minor intrinsic fluctuations. By comparison, the average His 101-Phe distances in UP1 ${ }^{\mathrm{dm}}$ increase to $16.3 \AA$, whereas the average Arg92-Phe distances increase to $17.2 \AA$. Clearly, UP1 ${ }^{\mathrm{dm}}$ experiences larger amplitude motions under identical simulation conditions, suggesting the salt bridge mutations destabilize the protein. The positional changes of the nucleobase pocket residues of UP1 ${ }^{\mathrm{dm}}$ are accompanied by other structural rearrangements throughout the protein as indicated by the pairwise rmsd for backbone atoms of $4.4 \AA$ (residues 11-179). In agreement with the structural transitions observed in the MD simulations, ${ }^{1} \mathrm{H}^{-15} \mathrm{~N}$ HSQC spectra of UP $1^{\mathrm{dm}}$ and wild type UP1 show significant chemical shift perturbations within RRM1 and the inter-RRM linker; sparse perturbations are also observed in RRM2 (Figure 6D and S8). Taken together, we conclude the salt-bridge interactions, which are located on the alpha helical side of the RNA binding surface, help stabilize the interRRM linker in a geometry poised for high affinity and specific RNA recognition.

\section{Discussion}

The RNA binding properties of hnRNP A1 have been studied extensively; yet no clear description has emerged as to how this protein interacts with its diverse RNA targets. Many of these targets fold into complex structures where only a few nucleobases are exposed for recognition. Chemical protection studies and NMR experiments have shown hnRNP A1 binds discrete sites within stem loops; thus indicating the protein does not bind folded RNA structures in an extended conformation as seen in the UP1-DNA complex (Figure S1). Since the RNA binding platform of canonical RRM domains typically recognize about 4 nucleotides, it has been difficult to understand how hnRNP A1 uses both RRMs to bind RNA stem loops specifically and with high affinity. The molecular recognition problem is further complicated because the RRM domains are fixed in an antiparallel orientation. Here, we show that only the RRM1 domain and interRRM linker interact with the apical loop of HIV SL3 ${ }^{\text {ESS3 }}$. We further demonstrated the R75:D155 and R88:D157 salt bridge interactions play a significant role in positioning the inter-RRM linker in a conformation primed to direct specific and high affinity RNA binding. The collective data presented opens a very intriguing question; can binding of RNA through RRM1 and the inter-RRM linker allosterically control hnRNP A1?

Comparison of the UP1-(AGU) structure to the unbound solution NMR structure (model 1 of pdb code $2 \mathrm{LYV}$ ) reveals UP1 undergoes a conformational change upon binding the 5'-AGU-3'trinucleotide (Figure 7A). The overall pairwise backbone rmsd between the 
two structures is $2.77 \AA$ (residues 11-179). Alignments of the individual domains and inter-RRM linker show the structural differences manifest primarily at the linker and RRM2: $0.67 \AA$ (RRM1; 11-88), $1.31 \AA$ (RRM2; 106-179) and $1.33 \AA$ (inter-RRM linker; 89-105). Given the greater similarities between the RRM1 domains, we conclude binding of the 5'-AGU-3' trinucleotide induces a conformational change that transmits across the inter-RRM linker to RRM2 (Figure 7A). In the unbound structure, His101 packs tightly against Phe17 leaving no available space to accommodate RNA (Figure 7B). Binding of rA within the nucleobase pocket requires that His 101 shift laterally away from Phe17 by $\sim 3.5 \AA$ (Figure 7B). Of note, the $\sim 3.5 \AA$ shift is outside the experimental resolution of the NMR ensemble (heavy atom rmsd $=1.22 \pm 0.22 \AA$ ), indicating the observed local change in conformation is significant. This local change triggers global structural rearrangements throughout RRM2 such that the entire domain moves away from RRM1 (Figure 7A). Interestingly, the rA N6 amino group forms a hydrogen bond to the $\alpha$ carbonyl oxygen of Arg88 (Figure 7C). This interaction establishes a direct and specific network where occupancy of an adenine within the first position of the nucleobase pocket communicates to RRM2 via the R88:D157 salt bridge. Indeed, the C-terminal end of helix 2 from RRM2 pivots away from RRM1 in the bound structure (Figure 7A). Larger movements of helix 2 are likely restricted due to strong coupling at the inter-RRM interface ${ }^{31}$.

The importance of the salt-bridge interactions to organize the relative positions of the RRM domains have been alluded to in earlier structural studies of UP1 ${ }^{4 ; 5 ; 7}$; however, the contributions of these interactions to the RNA binding properties were not determined. As described above, the R75D/R88D UP ${ }^{\mathrm{dm}}$ binds $\mathrm{SL}{ }^{\mathrm{ESS} 3} \sim 18$ fold weaker than wild type UP1 (Figure S6). The reduction in affinity stems from a very large decrease in total binding enthalpy (Figure 6B). This result was unexpected as the mutations were introduced on the alpha helical side of the RNA binding surface. Our MD simulations revealed the mutations lead to a breakdown of the nucleobase pocket by destabilizing the inter-RRM linker. The distorted pocket (Figure 6C) would fail to support stacking interactions with $\mathrm{rA}$ and $\mathrm{rG}$ as seen in the UP1-(AGU) crystal structure. Furthermore, rA would not trigger the conformational change in UP $1^{\mathrm{dm}}$ as observed for the wild type protein since His101 has moved $\sim 15 \AA$ away and no longer stacks with Phe17. The loss of these interactions might explain the large decrease in total binding enthalpy for the $\mathrm{UP} 1^{\mathrm{dm}}-\mathrm{SL} 3^{\mathrm{ESS} 3}$ complex. Consistent with our results, Mayeda et al reported the linker is important for the functional properties of hnRNP A1 since a deletion construct showed impaired alternative splicing activity and reduced affinity for a consensus RNA target ${ }^{6}$. The importance of the linker is further reflected by its phylogenetic conservation and because isolated RRM1 and RRM2 bind target RNA elements $\sim 100$ fold weaker than intact UP1 ${ }^{21 ; 32}$. Taken together, we conclude the salt bridge interactions organize the inter-RRM linker in a unique stereochemical environment, which enables specific and high affinity RNA recognition within the nucleobase pocket.

The rA N6 to Arg88 O hydrogen bond might also explain the AGU rich sequence preference observed in genome-wide CLIP studies and the ability of hnRNP Al to proofread 3' splice sites containing AG but not CG dinucleotides ${ }^{33 ; 34 ; 35}$. Cytidine is the only other nucleobase that would most readily satisfy this hydrogen bond interaction; however, the A-to-C substitution at position five of the SL3 ${ }^{\mathrm{ESS3}}$ apical loop had the 
largest reduction in binding affinity (Figure 5B and Table 2). Thus, we conclude the majority of the binding free energy originates from favorable stacking interactions of $\mathrm{rA}$ between His101 and Phe17. While a cytidine has the capacity to hydrogen bond with the carbonyl oxygen of Arg88, occupancy of a cytidine at this position might fail to induce a similar conformational change as observed with adenine. In consideration, the $\sim 10$ fold reduction in binding affinity observed for the A-to-C SL3 ${ }^{\mathrm{ESS} 3}$ mutant derives from a slower second order association rate constant (Table 2), which likely reflects a kinetic barrier to forming a stable complex.

Of note, our bound structure shows better agreement with the RRM domains of the free UP1 crystal structure ( $0.45 \AA$ rmsd for residues $11-88$ and 106-179), despite both having crystallized in different space groups ( $\mathrm{P} 12{ }_{1} 1$ free and $\mathrm{C} 222_{1}$ bound). Electron density for the inter-RRM linker was not observed in the free crystal structure, which precludes a detailed comparison of likely conformational changes exerted by RNA binding. It has been suggested that crystal lattice effects might cause non-native relative RRM orientations in UP $1^{31}$. This conclusion was reached largely based on the similarities between the free NMR structure and the DNA bound structure, which crystallized as a dimer. The overall backbone rmsd between our structure and the DNA bound structure is $2.78 \AA$ (residues 11-179), where the major changes occur in the orientation of the RRM2 domain. These differences likely stem from dimerization and the elongated DNA strand used in those studies ${ }^{7}$. For reasons discussed above, we believe the differences observed between our structure and the solution NMR structure represents an authentic RNA induced conformational change. Certainly, His 101 must reposition to create space for the RNA to bind and that change triggers other movements within the linker and throughout RRM2.

In summary, we have solved the first high-resolution structure of an hnRNP A1 domain bound to RNA and used the structure along with other biophysical data to determine a SAXS-scored structural model of the intact UP1-SL3 ${ }^{\mathrm{ESS} 3}$ complex. The model reveals a mechanism for how hnRNP A1 binds folded RNA targets where only a few nucleobases are exposed (Figure 4B). Given the number of biologically important RNA stem loops known to bind hnRNP A1, we expect the structure will have broad and significant implications. Moreover, the univalent RNA occupancy observed here opens the possibility that hnRNP A1 could be allosterically regulated.

\section{Materials and Methods}

\section{RNA Preparation}

$\mathrm{SL} 3^{\mathrm{ESS} 3}$ and cytosine-substituted constructs were in vitro transcribed and purified as previously described ${ }^{22}$. RNA concentrations were determined using theoretical molar extinction coefficients calculated by NanoDrop ${ }^{\mathrm{TM}} 2000$ software (Thermo Fisher).

Preparation of the UP1-SL3 ${ }^{\text {ESS }}$ complex 
The UP1 protein was prepared as a C-terminal His-tag fusion following a previously published protocol ${ }^{22}$. The UP1-SL3 ${ }^{\mathrm{ESS} 3}$ complex was formed by stepwise titrations of $\mathrm{SL}^{\mathrm{ESS} 3}$ into UP1. Complex formation was monitored by size exclusion chromatography on a Superdex 200 10/300 GL column (GE Healthcare Life Sciences). Peaks corresponding to a 1:1 UP1-SL3 ${ }^{\mathrm{ESS} 3}$ complex were collected and concentrated using an Amicon ultrafiltration device. As reported previously, identical and well-resolved peaks are observed on the SEC column for the UP1- SL3 ${ }^{\mathrm{ESS} 3}$ complex and unbound SL3 ${ }^{\mathrm{ESS} 3} 22$.

\section{Crystallization of UP1- (AGU)}

For crystallization, the UP1- SL3 ${ }^{\mathrm{ESS} 3}$ complex at $4.3 \mathrm{mg} / \mathrm{mL}$ in $10 \mathrm{mM}$ potassium phosphate $\mathrm{pH} 6.5,120 \mathrm{mM}$ potassium chloride, and $0.5 \mathrm{mM}$ EDTA was screened against the Hampton Natrix Screen using a Gryphon (Art Robbins Instruments). While under vapor diffusion, sitting drops containing $0.75 \mathrm{uL}$ of the complex and $0.25 \mathrm{uL}$ of well solution were incubated at $4{ }^{\circ} \mathrm{C}$. After one month, crystals were observed in condition A2 (2.5 $\mathrm{M}$ ammonium sulfate, $50 \mathrm{mM}$ MES $\mathrm{pH} 5.6$, and $10 \mathrm{mM}$ magnesium acetate). Crystals were cryoprotected in well solution with the addition of $20 \%$ glycerol and flash cooled in liquid nitrogen prior to data collection.

\section{Data Collection and Structure Determination}

Data were collected at LS-CAT at Advanced Photon Source at Argonne National Laboratory, beamline 21-ID-G and processed with HLK2000 ${ }^{36}$. The UP1-(AGU) complex crystallized in space group C222 ${ }_{1}$, with unit cell dimensions of $\mathrm{a}=40.557 \AA$, b $=64.523 \AA, c=141.352 \AA$ and $90^{\circ}$ for all angles and with 1 molecule in the asymmetric unit. The structure was solved by molecular replacement with Phaser ${ }^{37}$, using the apo structure of UP1 (pdb code 1L3K) as a search model. The initial Fo-Fc electron density map (Figure 1D) indicated the presence of RNA in the RRM1 binding site. The other 24 residues of the SL3 ${ }^{\mathrm{ESS} 3}$ structure were missing due to possible RNase hydrolysis, which is consistent with the calculated solvent content of $40 \%$ for UP1 alone. The structure was fit to the electron density in $\operatorname{Coot}^{38}$ and refined to $1.92 \AA$ using Buster ${ }^{39}$, then validated with Molprobity ${ }^{40}$. All residues were found to be in the allowed regions of the Ramachandran plot with the exception of residues $1-7$ and $189-196$, which were disordered in the structure. Data refinement and statistics are given in Table 1.

SAXS data collection and molecular reconstructions

SEC-SAXS (Size Exclusion Chromatography - Small Angle X-ray Scattering) experiments were performed on a 1.2:1 UP1-SL3 ${ }^{\mathrm{ESS} 3}$ complex at BioCAT (beamline 18ID, APS). Complex samples were prepared as described above. The basic setup for SECSAXS was based on work described by Mathews $e t a l^{41}$. The camera included a focused $12 \mathrm{KeV}(1.03 \AA) \mathrm{x}$-ray beam, a $1.5 \mathrm{~mm}$ quartz capillary sample cell, a sample detector distance of $\sim 3.5 \mathrm{~m}$ and a Pilatus $31 \mathrm{M}$ (Dectris) detector. The q-range was $\sim 0.005-0.3$ $\AA^{-1}$. In order to ensure sample monodispersity, we used an in-line SEC setup, which included an AKTA-pure FPLC unit and Superdex 200 10/300 GL column (GE Healthcare Life Sciences). The column outlet was directly connected to the SAXS sample 
cell. One second exposures were collected every 2 seconds during the gel filtration chromatography run. Exposures immediately before and/or after the elution of the sample (co-incident with the UV peak on the chromatogram) were treated as complex plus buffer curves. Data were corrected for background scattering by subtracting the buffer curve from the complex plus buffer curves.

Molecular reconstructions were performed in Primus ${ }^{42}$ from the ATSAS $^{43}$ suite of programs. In preparation for envelope determination, the scattering curve was analyzed by Guinier fitting to test for aggregation and estimate the $\operatorname{Rg}\left(\mathrm{R}_{\mathrm{g}} \bullet \mathrm{q}<1.3\right)$, the scattering curve was fit for molecular reconstruction and the $\mathrm{p}(\mathrm{r})$ distance distribution plot was generated using $\mathrm{GNOM}^{44}$. Ab initio models were constructed using DAMMIF ${ }^{27 ; 28}$, generating ten independent models. The models were then aligned and averaged using $\mathrm{DAMAVER}^{28}$, and the most probable model was generated from the averaged model using damfilt.

Data-driven structural modeling and SAXS filtering

Structural docking calculations using HADDOCK 2.2 $23 ; 24$ were performed on the WeNMR Grid ${ }^{45}$. The UP1-(AGU) crystal and first model of the SL3 ${ }^{\mathrm{ESS} 3}$ NMR structure (PDB code 2LYV) were used for docking. In preparation for the calculations, the AGU trinucleotide and solvent molecules were removed from the crystal structure to allow for docking of SL3 ${ }^{\mathrm{ESS} 3}$.

To guide the docking, active residues were defined using interactions derived from the UP1-(AGU) crystal structure along with saturation transfer difference $\mathrm{NMR}^{22},{ }^{1} \mathrm{H}_{-}{ }^{13} \mathrm{C}$ CSPs between ${ }^{13} \mathrm{C}$-labeled SL3 ${ }^{\mathrm{ESS} 3}$ constructs and unlabeled UP1 (Figure 1C), and thermodynamic perturbations derived from calorimetric titrations of the SL3 ${ }^{\mathrm{ESS} 3}$ apical loop mutants with UP1 ${ }^{22}$. The HADDOCK program was set to automatically detect passive residues along with regions for semi-flexible refinement. Initially, 1000 rigid body structures were calculated. The 200 most favorable structures then underwent semiflexible and water refinement. The resulting structures were then clustered according to a $7.5 \AA$ RMSD cutoff with a minimum cluster size of four.

The resulting HADDOCK clusters were then filtered against the SAXS data. The scattering curve of each structure was calculated with CRYSOL ${ }^{29}$ using 256 points for the theoretical curve, constant subtraction and a maximum scattering vector of $0.25 \AA^{-1}$. The average $\chi^{2}$ of each cluster was then used to remove clusters that fit the experimental data poorly $\left(\chi^{2}>1.3\right)$. The five best scoring $\left(\chi^{2}\right)$ structures of the remaining clusters were then compared to the SAXS ab initio molecular reconstruction using SUPCOMB $20^{30}$. Clusters were then ranked according to average NSD values and best-fit structural models were selected based on the lowest NSD value.

Unfolded SL3 ${ }^{\mathrm{ESS} 3}$ was prepared for HADDOCK simulation through generation of an unfolded pdb template using XPLOR-NIH ${ }^{46}$. The unfolded $\mathrm{SL}_{3}{ }^{\mathrm{ESS}}{ }^{3}$ strand was then relaxed using the ff99bsc0_chiOL3 force field in Amber 12 to allow for relaxation of the rigid oligonucleotide template. The unfolded SL3 ${ }^{\mathrm{ESS} 3}$ construct was solvated in a TIP3P 

minimization, the temperature was increased from 0 to $300 \mathrm{~K}$ over $40 \mathrm{ps}$ and the system was simulated for 500 ps at $300 \mathrm{~K}$ for relaxation..

\section{Preparation of UP1 and UPI ${ }^{d m}$ for NMR analysis}

The UP1 protein was prepared as a N-terminal His-tag fusion, with a TEV cleavage sequence located at the C-terminal end of the His-tag. This recombinant protein was overexpressed and purified with a $\mathrm{Ni}^{2+}$ column as previously described ${ }^{21}$. The His-tag was cleaved from UP1 by addition of TEV protease overnight at $4{ }^{\circ} \mathrm{C}$ in cleavage buffer (120 mM KCl, $10 \mathrm{mM} \mathrm{KH}_{2} \mathrm{PO}_{4} \mathrm{pH}=6.5,10 \mathrm{mM} \mathrm{NaCl}, 0.5 \mathrm{mM}$ EDTA, $20 \mathrm{mM}$ imidazole and $1 \mathrm{mM}$ DTT). Cleaved UP1 was run back through the $\mathrm{Ni}^{2+}$ column with the flow through collected. It was further purified through gel filtration chromatography on the FPLC as previously described ${ }^{22}$. All NMR experiments were prepared, performed, and processed as previously described ${ }^{31}$.

\section{Biolayer Interferometry Kinetic Binding Studies}

UP1 protein and SL3 ${ }^{\mathrm{ESS} 3}$ RNA (WT and mutants) were produced as described above. The C-terminal UP1-(His) 6 construct was used for all kinetic experiments. UP1 was biotinylated using EZ-Link NHS-PEG4-Biotin (Thermo Fisher Scientific) at a 1:1 protein to biotin ratio to prevent over-biotinylation. Excess biotin was then removed via dialysis into the buffer used for kinetic experiments $\left(120 \mathrm{mM} \mathrm{KCl}, 10 \mathrm{mM} \mathrm{K}_{2} \mathrm{HPO}_{4}\right.$ and $0.5 \mathrm{mM}$ EDTA at $\mathrm{pH}$ 6.5). Concentrated RNA samples were diluted into kinetic buffer and serial dilutions into 96 well plates were performed to account for the observed $\mathrm{K}_{\mathrm{D}}$ values.

UP1-SL3 ${ }^{\text {ESS3 }}$ kinetic experiments were conducted in triplicate using an Octet Red 96 instrument (FortéBio). All kinetic experiments were performed at $25^{\circ} \mathrm{C}$ while stirred at $1000 \mathrm{rpm}$ to create a homogeneous mixture. The biotinylated UP1 was loaded onto Super Streptavidin Biosensors (FortéBio) via offline loading. The experiments were performed as follows: after initial equilibration in buffer for $600 \mathrm{~s}$, the baseline was stabilized for 60 s, RNA was associated for 300s, and the RNA was dissociated for 900s The process of baseline, association and dissociation was repeated for all serial dilutions. FortéBio Octet Data Acquisition and Analysis Software version 7.0 was utilized to perform initial data acquisition and processing and GraphPad Prism 6.0 was used to fit the kinetic data (Figure S5).

Analysis of the BLI rate parameters was done following a similar procedure as described by Ramos $e t a l^{47}$. In brief, global fitting of the dissociation rate profiles (Figure S5) were performed using equation 1 to obtain first order off rate constants $\left(\mathrm{k}_{\mathrm{off}}\right)$. Global fits take into account experimental variability of the individual dissociation profiles and therefore provide a robust estimate of the rate constants. Reduced $\chi^{2}$ values $<1$ were obtained for global fitting the experimental dissociation data to equation 1.

(1) $\mathrm{Y}=\left(\mathrm{Y}_{\mathrm{o}}-\mathrm{NS}\right)^{*} \exp ^{-\mathrm{kt}}+\mathrm{NS}$ 
$\mathrm{Y}_{\mathrm{o}}$ represents binding at time zero and NS is binding (nonspecific) at infinite time. Steady-state binding isotherms were constructed by plotting the amplitude $(\Delta$ Signal $=$ Signal $_{\mathrm{t}=0 \mathrm{~s}}-$ Signal $_{\mathrm{t}=500 \mathrm{~s}}$ ) from the dissociation curves against SL3 ${ }^{\mathrm{ESS}}$ concentration. The dissociation constants $\left(\mathrm{K}_{\mathrm{D}}\right)$ were derived by fitting the steady-state binding curves to a 1:1 Langmuir isotherm shown in equation 2.

(2) $\mathrm{Y}=\mathrm{B}_{\max }[\mathrm{ESS} 3] / \mathrm{K}_{\mathrm{D}}+[\mathrm{ESS} 3]$

The bimolecular association rate constants $\left(\mathrm{k}_{\mathrm{on}}\right)$ were then calculated from equation 3 .

(3) $\mathrm{K}_{\mathrm{D}}=\mathrm{k}_{\mathrm{off}} / \mathrm{k}_{\mathrm{on}}$.

The kinetic parameters reported in Table 2 correspond to averages of three independent replicates. Of note, control experiments using biotinylated SL3 ${ }^{\mathrm{ESS} 3}$ (purchased from IDT) immobilized to the sensor surface gave comparable kinetic parameters (data not shown), indicating biotinylation of UP1 does not affect the interaction.

\section{Calorimetric Titrations}

Titrations were conducted at $25{ }^{\circ} \mathrm{C}$ using a VP-ITC calorimeter (MicroCal, LLC). The Cterminal UP1-(His) 6 construct was used for all titrations. The RNAs were initially resuspended in water and annealed by heating the samples at $95{ }^{\circ} \mathrm{C}$ for 2 minutes and snap cooling on ice. The annealed RNA samples and UP1 protein were then individually exchanged into buffer $\left(120 \mathrm{mM} \mathrm{KCl}, 10 \mathrm{mM} \mathrm{K}_{2} \mathrm{HPO}_{4}, 1 \mathrm{mM}\right.$ TCEP and $0.5 \mathrm{mM}$ EDTA at $\mathrm{pH}$ 6.5) using Amicon Ultra $3 \mathrm{~K}$ centrifugal filters. The RNA was diluted to concentrations ranging from $2-2.5 \mu \mathrm{M}$ while the protein was diluted between 40 to 45 $\mu \mathrm{M}$. UP1 was titrated into $1.4 \mathrm{~mL}$ of RNA over 35 injections $(8 \mu \mathrm{L}$ each), where both RNA titrations were performed in three replicates (Figure S3). Data fitting was performed in Origin version 7.0, where the raw data was fit with nonlinear least-squares fitting after corrections for dilution were performed by subtracting the average heats from the last few points of the saturated upper asymptotes.

\section{Molecular Dynamic Simulations}

To study the effects of the double salt bridge mutation on UP1 structure, a $10 \mathrm{~ns}$ simulation using the ff12SB force field was conducted with the first model of the unbound NMR structure of UP1 (pdb code 2LYV) in Amber 12 ${ }^{48}$. The R75D R88D double mutant was created using the mutation function in $\mathrm{PyMol}^{49}$ from the wild type UP1 construct. Using xLeap, both constructs were neutralized with $\mathrm{K}^{+}$and $\mathrm{Cl}^{-}$ions and solvated in a TIP3P octahedron of water with no less than $8.0 \AA$ between all atoms and the edge of the water octahedron. This produced a wild type and double mutant system consisting of $\sim 8900$ water molecules each.

The water molecules of the octahedron were first minimized by steepest descent for 500 steps followed by 500 steps of conjugate gradient descent. During this stage, the protein was held fixed with a force constant of $500 \mathrm{kcal} \mathrm{mol}^{-1} \AA^{-2}$, periodic boundaries were 
activated and non-bonded interactions greater than $10 \AA$ were not calculated. After solvent minimization, the unrestrained system was minimized with 2000 steps of steepest descent followed by 2000 steps of conjugate gradient descent with parameters otherwise identical to that of the first minimization.

The two explicitly solvated systems were then subjected to heating from 0 to $300 \mathrm{~K}$ over 20,000 steps of 2 fs each ( $40 \mathrm{ps}$ total). During this stage, minimization was turned off and the protein was kept fixed with positional restraints of $10 \mathrm{kcal} \mathrm{mol}^{-1} \AA^{-2}$. SHAKE was activated to constrain bonds involving hydrogen, a non-bonded cutoff of $10 \AA$ was used, constant volume periodic boundaries were activated and Langevin Dynamics with a collision frequency of $1 \mathrm{ps}^{-1}$ was used for temperature control.

Lastly, the entire system was subjected to simulation at $300 \mathrm{~K}$ for $10 \mathrm{~ns}$ using PMEMD. During this stage, Langevin dynamics at a collision frequency of $2 \mathrm{ps}^{-1}$ was used for temperature control along with a constant pressure periodic boundary with an average pressure of $1 \mathrm{~atm}$, isotropic position scaling and a 2 ps pressure relaxation time. Nonbonded interactions larger than $10 \AA$ were ignored and SHAKE was used to constrain bonds involving hydrogen. The systems were simulated over 5,000,000 steps of $2 \mathrm{fs}$ each. Restart files were written every $100 \mathrm{ps}$ while the trajectory was written every 5 ps. The resulting structures of the wild type and R75D R88D double mutant were visualized in PyMol and the trajectories were analyzed using cpptraj.

\section{Accession numbers}

PDB coordinates and structure factors of the UP1-(AGU) complex have been deposited in the Protein Data Bank under accession number 4YOE.

\section{Acknowledgements}

This work was supported by National Institutes of Health Grants R01GM101979 (BST) and P50GM103297 (JAS). Use of the Advanced Photon Source, an Office of Science User Facility operated for the U.S. Department of Energy (DOE) Office of Science by Argonne National Laboratory, was supported by the U.S. DOE under Contract No. DEAC02-06CH11357. Use of the LS-CAT Sector 21 was supported by the Michigan Economic Development Corporation and the Michigan Technology Tri-Corridor (Grant 085P1000817). We would like to thank Dr. David Smith at LS-CAT for assistance with crystal screening and remote data collection. The authors thank Srinivas Chakravarthy (APS ID18 BioCAT) for his assistance with SEC-SAXS data collection and preliminary analysis. Use of the Pilatus $31 \mathrm{M}$ detector was provided by grant 1S10OD018090-01 from NIGMS. 


\section{References}

1. He, Y. \& Smith, R. (2009). Nuclear functions of heterogeneous nuclear ribonucleoproteins A/B. Cell Mol Life Sci 66, 1239-56.

2. Jean-Philippe, J., Paz, S. \& Caputi, M. (2013). hnRNP A1: the Swiss army knife of gene expression. Int J Mol Sci 14, 18999-9024.

3. Siomi, H. \& Dreyfuss, G. (1995). A nuclear localization domain in the hnRNP A1 protein. J Cell Biol 129, 551-60.

4. Shamoo, Y., Krueger, U., Rice, L. M., Williams, K. R. \& Steitz, T. A. (1997). Crystal structure of the two RNA binding domains of human hnRNP A1 at $1.75 \mathrm{~A}$ resolution. Nat Struct Biol 4, 215-22.

5. $\quad \mathrm{Xu}, \mathrm{R}$. M., Jokhan, L., Cheng, X., Mayeda, A. \& Krainer, A. R. (1997). Crystal structure of human UP1, the domain of hnRNP A1 that contains two RNArecognition motifs. Structure 5, 559-70.

6. Mayeda, A., Munroe, S. H., Xu, R. M. \& Krainer, A. R. (1998). Distinct functions of the closely related tandem RNA-recognition motifs of hnRNP A1. RNA 4, 1111-23.

7. Ding, J., Hayashi, M. K., Zhang, Y., Manche, L., Krainer, A. R. \& Xu, R. M. (1999). Crystal structure of the two-RRM domain of hnRNP A1 (UP1) complexed with single-stranded telomeric DNA. Genes \& development 13, 1102-15.

8. Saliou, J. M., Bourgeois, C. F., Ayadi-Ben Mena, L., Ropers, D., Jacquenet, S., Marchand, V., Stevenin, J. \& Branlant, C. (2009). Role of RNA structure and protein factors in the control of HIV-1 splicing. Frontiers in bioscience : a journal and virtual library 14, 2714-29.

9. Guil, S. \& Caceres, J. F. (2007). The multifunctional RNA-binding protein hnRNP A1 is required for processing of miR-18a. Nat Struct Mol Biol 14, 591-6.

10. Michlewski, G. \& Caceres, J. F. (2010). Antagonistic role of hnRNP A1 and KSRP in the regulation of let-7a biogenesis. Nature structural \& molecular biology 17, 1011-8.

11. Michlewski, G., Guil, S. \& Caceres, J. F. (2011). Stimulation of pri-miR-18a Processing by hnRNP A1. Advances in experimental medicine and biology 700, 28-35.

12. Choudhury, N. R. \& Michlewski, G. (2012). Terminal loop-mediated control of microRNA biogenesis. Biochem Soc Trans 40, 789-93.

13. Castilla-Llorente, V., Nicastro, G. \& Ramos, A. (2013). Terminal loop-mediated regulation of miRNA biogenesis: selectivity and mechanisms. Biochem Soc Trans 41, 861-5.

14. Lin, J. Y., Shih, S. R., Pan, M., Li, C., Lue, C. F., Stollar, V. \& Li, M. L. (2009). hnRNP A1 interacts with the 5' untranslated regions of enterovirus 71 and Sindbis virus RNA and is required for viral replication. $J$ Virol 83, 6106-14.

15. Shih, S. R., Stollar, V. \& Li, M. L. (2011). Host factors in enterovirus 71 replication. $J$ Virol 85, 9658-66.

16. Levengood, J. D., Tolbert, M., Li, M. L. \& Tolbert, B. S. (2013). High-affinity interaction of hnRNP A1 with conserved RNA structural elements is required for translation and replication of enterovirus 71. RNA Biol 10, 1136-45. 
17. Marchand, V., Mereau, A., Jacquenet, S., Thomas, D., Mougin, A., Gattoni, R., Stevenin, J. \& Branlant, C. (2002). A Janus splicing regulatory element modulates HIV-1 tat and rev mRNA production by coordination of hnRNP A1 cooperative binding. J Mol Biol 323, 629-52.

18. Tange, T. O., Damgaard, C. K., Guth, S., Valcarcel, J. \& Kjems, J. (2001). The hnRNP A1 protein regulates HIV-1 tat splicing via a novel intron silencer element. EMBO J 20, 5748-58.

19. Damgaard, C. K., Tange, T. O. \& Kjems, J. (2002). hnRNP A1 controls HIV-1 mRNA splicing through cooperative binding to intron and exon splicing silencers in the context of a conserved secondary structure. $R N A \mathbf{8}, 1401-15$.

20. Michlewski, G., Guil, S., Semple, C. A. \& Caceres, J. F. (2008). Posttranscriptional regulation of miRNAs harboring conserved terminal loops. Mol Cell 32, 383-93.

21. Levengood, J. D., Rollins, C., Mishler, C. H., Johnson, C. A., Miner, G., Rajan, P., Znosko, B. M. \& Tolbert, B. S. (2011). Solution Structure of the HIV-1 Exon Splicing Silencer 3. Journal of Molecular Biology 415, 680-698.

22. Rollins, C., Levengood, J. D., Rife, B. D., Salemi, M. \& Tolbert, B. S. (2014). Thermodynamic and Phylogenetic Insights into hnRNP A1 Recognition of the HIV-1 Exon Splicing Silencer 3 Element. Biochemistry 53, 2172-84.

23. de Vries, S. J., van Dijk, M. \& Bonvin, A. M. (2010). The HADDOCK web server for data-driven biomolecular docking. Nature protocols 5, 883-97.

24. Karaca, E., Melquiond, A. S., de Vries, S. J., Kastritis, P. L. \& Bonvin, A. M. (2010). Building macromolecular assemblies by information-driven docking: introducing the HADDOCK multibody docking server. Molecular \& cellular proteomics : MCP 9, 1784-94.

25. Martin-Tumasz, S., Richie, A. C., Clos, L. J., 2nd, Brow, D. A. \& Butcher, S. E. (2011). A novel occluded RNA recognition motif in Prp24 unwinds the U6 RNA internal stem loop. Nucleic Acids Res 39, 7837-47.

26. Hennig, J., Wang, I., Sonntag, M., Gabel, F. \& Sattler, M. (2013). Combining NMR and small angle X-ray and neutron scattering in the structural analysis of a ternary protein-RNA complex. J Biomol NMR 56, 17-30.

27. Dimitri, I., \& Svergun, DF. (2009). DAMMIF, a program for rapid ab-initio shape determination in small-angle scattering. Journal of Applied Crystallography 43, 342-346.

28. Volkov, V., \& Svergun, DI. (2003). Uniqueness of ab initio shape determination in small-angle scattering. Journal of Applied Crystallography 36, 860-864.

29. Svergun, D., Barberato, C, \& Koch, MHJ. (1995). CRYSOL - a Program to Evaluate X-ray Solution Scattering of Biological Macromolecules from Atomic Coordinates. Journal of Applied Crystallography 28, 768-773.

30. Kozin, M., \& Svergun, DI. (2001). Automated matching of high- and lowresolution structural models. Journal of Applied Crystallography 34, 33-41.

31. Barraud, P. \& Allain, F. H. (2013). Solution structure of the two RNA recognition motifs of hnRNP A1 using segmental isotope labeling: how the relative orientation between RRMs influences the nucleic acid binding topology. J Biomol NMR 55, 119-38. 
32. Shamoo, Y., Abdul-Manan, N., Patten, A. M., Crawford, J. K., Pellegrini, M. C. \& Williams, K. R. (1994). Both RNA-binding domains in heterogenous nuclear ribonucleoprotein A1 contribute toward single-stranded-RNA binding. Biochemistry 33, 8272-81.

33. Tavanez, J. P., Madl, T., Kooshapur, H., Sattler, M. \& Valcarcel, J. (2012). hnRNP A1 proofreads 3' splice site recognition by U2AF. Mol Cell 45, 314-29.

34. Han, K., Yeo, G., An, P., Burge, C. B. \& Grabowski, P. J. (2005). A combinatorial code for splicing silencing: UAGG and GGGG motifs. PLoS Biol 3, e158.

35. Huelga, S. C., Vu, A. Q., Arnold, J. D., Liang, T. Y., Liu, P. P., Yan, B. Y., Donohue, J. P., Shiue, L., Hoon, S., Brenner, S., Ares, M., Jr. \& Yeo, G. W. (2012). Integrative genome-wide analysis reveals cooperative regulation of alternative splicing by hnRNP proteins. Cell Rep 1, 167-78.

36. Otwinowski, Z. \& Minor, W. (1997). Processing of X-ray diffraction data collected in oscillation mode. Methods in Enzymology 276, 307-327.

37. McCoy, A. J., Grosse-Kunstleve, R. W., Adams, P. D., Winn, M. D., Storoni, L. C. \& Read, R. J. (2007). Phaser crystallographic software. J Appl Crystallogr 40, 658-674.

38. Emsley, P. \& Cowtan, K. (2004). Coot: model-building tools for molecular graphics. Acta Crystallogr D Biol Crystallogr 60, 2126-32.

39. Roversi P, S. A., Smart OS, Vonhein C, Womack TO. (2011). BUSTER version 2.11.2. Global Phasing Lts., Cambridge, United Kingdom.

40. Davis, I. W., Leaver-Fay, A., Chen, V. B., Block, J. N., Kapral, G. J., Wang, X., Murray, L. W., Arendall, W. B., 3rd, Snoeyink, J., Richardson, J. S. \& Richardson, D. C. (2007). MolProbity: all-atom contacts and structure validation for proteins and nucleic acids. Nucleic Acids Res 35, W375-83.

41. Mathew, E., Mizra, A, \& Menhart, N. (2004). Liquid-chromatography-coupled SAXS for accurate sizing of aggregating proteins. $J$ Synchrotron Radiat 11, 314318.

42. Konarev, P., Volkov, VV, Sokolova, AV, Koch, MH, \& Svergun, DI. (2003). PRIMUS: a Windows PC-based system for small- angle scattering data analysis. Journal of Applied Crystallography 36, 1277-1282.

43. Petoukhov, M., Franke, D, Shkumatov, AV, Tria, G, Kikhney, AG, Gajda, M, Gorba, C, Mertens, H, Konarev, PV, \& Svergun, DI. (2012). New developments in the ATSAS program package for small-angle scattering data analysis. Journal of Applied Crystallography 45, 342-350.

44. Semenyuk, A., \& Svergun, DI. (1991). GNOM-a program package for smallangle scattering data processing. Journal of Applied Crystallography 24, 537-540.

45. de Vries, S. J., van Dijk, M. \& Bonvin, A. M. (2010). The HADDOCK web server for data-driven biomolecular docking. Nat Protoc 5, 883-97.

46. Schwieters, C. D., Kuszewski, J. J., Tjandra, N. \& Clore, G. M. (2003). The Xplor-NIH NMR molecular structure determination package. Journal of magnetic resonance 160, 65-73.

47. Nicastro, G., Garcia-Mayoral, M. F., Hollingworth, D., Kelly, G., Martin, S. R., Briata, P., Gherzi, R. \& Ramos, A. (2012). Noncanonical G recognition mediates KSRP regulation of let-7 biogenesis. Nat Struct Mol Biol 19, 1282-6. 
48. Case, D. A., Cheatham, T. E. I., Darden, T., Gohlke, H., Luo, R., Merz, K. M. J., Onufriev, A., Simmerling, C., Wang, B. \& Woods, R. (2005). The Amber biomolecular simulation programs. J. Computat. Chem. 26, 1668-1688.

49. DeLano Scientific. (2002). The PyMOL molecular graphics system. DeLano, W. L. 


\section{Figure Legends}

Figure 1. The UP1 domain of hnRNP A1 makes site-specific contacts with the HIV SL3 ${ }^{\text {ESS3 }}$ apical loop. A). Domain organization of human hnRNP A1 showing the Nterminal RNA recognition motifs (RRM1=yellow and RRM2=blue) and the C-terminal glycine-rich domain (gray). UP1 domain boundaries are defined based on crystal structure reported here. Signature RNP motifs are designated. B) Secondary structure of HIV SL3 ${ }^{\text {ESS3 }}$ as confirmed by NMR spectroscopy ${ }^{21}$. C) ${ }^{1} \mathrm{H}_{-}{ }^{13} \mathrm{C}$ HMQC detection of sitespecific interaction of UP1 with ${ }^{13} \mathrm{C}(\mathrm{A})$-labeled SL3 ${ }^{\mathrm{ESS} 3}$. Red C2-H2 correlation peaks correspond to free SL3 ${ }^{\mathrm{ESS} 3}$ and black peaks correspond to a 1.5:1 UP1-SL3 ${ }^{\mathrm{ESS} 3}$ complex. The blue box denotes $\mathrm{C} 2-\mathrm{H} 2$ correlation peaks from adenine bases engaged in $\mathrm{W}-\mathrm{C}$ base pairs. The spectra were collected at $298 \mathrm{~K}$ in $140 \mathrm{mM} \mathrm{K}^{+} \mathrm{pH} 6.5$. Note, the $\mathrm{N} 1$ imino group of $\mathrm{A} 8450$ titrates with a $\mathrm{pK}_{\mathrm{a}}$ of around 6.5 and therefore its $\mathrm{C} 2-\mathrm{H} 2$ correlation peak is exchanged broaden under the conditions of the binding studies.

Figure 2. Crystal structure of the UP1-(AGU) complex at $1.92 \AA$ resolution. A) Ribbon diagram of UP1 (RRM1=yellow; linker=gray; and RRM2=marine) bound to the 5'-AGU3' trinucleotide (shown as ball and sticks) to the RRM1 domain. B) RRM1 domain of UP1 bound to 5'-AGU-3' showing the omit (Fo-Fc) electron density map (green grid) of the RNA trinucleotide contoured at $3 \sigma$. C) Stereodiagram of the UP1-(AGU) binding interactions. Hydrogen bonds are shown as black dashed lines. Interacting residues shown as ball-n-sticks with 5'-AGU-3' carbons in gray, RRM1 carbons in yellow, linker carbons in dark gray, nitrogens in blue, oxygens in red and phosphorus in orange. D) Electrostatic surface potential of the nucleobase pocket. The surface is colored according to the calculated electrostatic potential from $-4 k T / e$ (red) to $4 k T / e$ (blue). The 5'-AGU3' trinucleotide is shown in ball-n-stick representation.

Figure 3. SEC-SAXS analysis of the UP1-SL3 ${ }^{\text {ESS3 }}$ complex. A) SAXS scattering profile of a 1.2:1 UP1-SL3 ${ }^{\mathrm{ESS} 3}$ complex collected at $298 \mathrm{~K}$ in $\mathrm{pH} 6.5$ phosphate buffer containing $140 \mathrm{mM} \mathrm{K}^{+}$. Scattering intensities have been buffer corrected. Inset shows the Guinier plot and calculated $\mathrm{R}_{\mathrm{g}}(25.02 \pm 2.81 \AA)$. The predicted MW based on the scattering intensity at zero angle, $\mathrm{I}(0)$, is $30.7 \mathrm{kDa}$, which is excellent agreement with the expected MW (33.8 kDa) of a 1:1 complex. B) Pair distance distribution of the complex providing $\mathrm{R}_{\mathrm{g}}$ of $\sim 26 \AA \mathrm{a} \mathrm{d}_{\max }$ of $93.5 \AA$. The distribution was calculated using $\mathrm{GNOM}^{44}$. C) $\mathrm{Ab}$ initio molecular reconstruction of the complex determined using DAMMIF/DAMAVER ${ }^{27 ;}$, providing a NSD value of $0.61 \pm 0.02$. The bead models were converted to molecular surfaces using $\mathrm{PyMol}^{49}$.

Figure 4. SAXS-scored structural model of the UP1-SL3 ${ }^{\mathrm{ESS} 3}$ complex. A) Representative structural model, derived from the best SAXS-scored cluster $\left(\chi^{2}=0.98 \pm 0.01\right)$, docked into the molecular reconstruction using SUPCOMB ${ }^{30}$. The NSD value is 0.80 indicating excellent agreement between the model and molecular reconstruction. B) Cartoon representation of the UP1-SL3 ${ }^{\mathrm{ESS} 3}$ complex revealing UP1 docks onto the apical loop (red) of SL3 ${ }^{\text {ESS3 }}$ through its RRM1 domain (yellow) and inter-RRM linker (gray). The RRM2 (blue) domain is more than $20 \AA$ away from the apical loop, where its beta sheet surface runs antiparallel to RRM1. The structural model also reveals potential 
electrostatic interactions between a $\mathrm{K} / \mathrm{R}$ cluster of RRM1 $\beta 2 / 3$ loop and the phosphodiester backbone.

Figure 5. BLI binding studies reveal rate parameters affected by SL3 ${ }^{\text {ESS3 }}$ apical loop substitutions. A) Representative (3 replicates) steady-state isotherms derived as described in materials and methods for each cytidine-substituted SL3 ${ }^{\mathrm{ESS} 3}$ construct. Kinetic studies were performed at $298 \mathrm{~K}$ in pH 6.5 phosphate buffer containing $140 \mathrm{mM} \mathrm{K}^{+}$. B) Log plot summarizing kinetic and thermodynamic parameters observed for UP1-SL3 ${ }^{\text {ESS3 }}$ interaction studies. The $\mathrm{K}_{\mathrm{D}}$ values measured by calorimetric titrations were reported previously $^{22}$.

Figure 6. Salt bridge interactions orient the inter-RRM linker to a unique stereochemical environment. A) Zoomed view of the R75:D155 and R88:D157 salt bridge interactions that couple RRM1 (yellow) and RRM2 (marine). R75 and R88 were both mutated to Asp residues in order to investigate the influence of the salt bridge interactions on RNA binding and inter-RRM structural integrity. B) Comparison of the thermodynamic profile of UP1 and UP $1^{\mathrm{dm}}$ titrations with SL3 ${ }^{\mathrm{ESS} 3}$. Thermodynamic parameters represent average +/- sd for 3 replicates. Calorimetric titrations were performed at $298 \mathrm{~K}$ in $\mathrm{pH} 6.5$ phosphate buffer containing $140 \mathrm{mM} \mathrm{K}^{+}$. C) MD simulations reveal the salt-bridge interactions are important to maintain the structural integrity of the nucleobase pocket environment. Top, starting structure of UP at $\mathrm{t}=0 \mathrm{~ns}$. The residues constituting the nucleobase pocket (Phe17, Phe57, Phe59, Arg92 and His101) are shown in stick representation. Average distances between $\mathrm{C} \alpha-\mathrm{C} \alpha$ positions of Arg92/His101 and Phe residues were used as a metric of the degree of structural change. Middle, wild type UP1 structure following $10 \mathrm{~ns}$ of simulation. Bottom, UP1 ${ }^{\mathrm{dm}}$ structure following $10 \mathrm{~ns}$ of simulation . D) Overlay of ${ }^{1} \mathrm{H}-{ }^{15} \mathrm{~N}$ HSQC spectra for wild type (blue) and UP ${ }^{\mathrm{dm}}$ (black). Labels denote a subset of wild type cross peaks that show significant perturbations in the double mutant.

Figure 7. Comparison of the UP1 solution NMR structure to the UP1-(AGU) structure reveals a conformational change. A) Backbone superimposition of the NMR structure (RRM1=light brown; linker=light gray; and RRM2=light purple) and the complex $(\mathrm{RRM} 1=$ =ellow; linker=gray; and $\mathrm{RRM} 2=$ blue). The structures are superimposed on the RRM1 domain (residues 11-89). The bottom view is rotated $90^{\circ}$ about the $\mathrm{x}$-axis from the top view. B) Zoomed view of the His101-Phe17 stacking interaction observed in the (left) NMR structure and (right) in the complex. Binding of the 5'-AGU-3' trinucleotide requires His 101 to shift laterally by $\sim 3.5 \AA$. C) Binding of $\mathrm{rA}$ within the nucleobase pocket establishes a direct network to RRM2 via the R88:D157 salt bridge interaction. 
Table 1: Crystallography Data Collection and Refinement Statistics

\begin{tabular}{|c|c|}
\hline \multicolumn{2}{|l|}{ Data Collection } \\
\hline SpaceGroup & $\mathrm{C} 222_{1}$ \\
\hline Unit Cell a, b, c $(\AA)$ & $\begin{array}{c}40.557,64.523 \\
141.352\end{array}$ \\
\hline Wavelength $(\AA)$ & 0.9786 \\
\hline Resolution $(\AA)^{1}$ & $1.92(1.95-1.92)$ \\
\hline $\operatorname{Rsym}(\%)^{2}$ & $7.1(46.3)$ \\
\hline$<\mathrm{I} / \sigma \mathrm{I}>^{3}$ & $20(3)$ \\
\hline Completeness $(\%)^{4}$ & $100(99.4)$ \\
\hline Redundancy & $13.9(11.0)$ \\
\hline \multicolumn{2}{|l|}{ Refinement } \\
\hline Resolution $(\AA)$ & 1.92 \\
\hline R-Factor $(\%)^{5}$ & 18.8 \\
\hline Rfree $(\%)^{6}$ & 23.7 \\
\hline Protein atoms & 1417 \\
\hline Nucleic Acid atoms & 62 \\
\hline Water Molecules & 90 \\
\hline Unique Reflections & 14587 \\
\hline \multicolumn{2}{|l|}{ R.m.s.d. ${ }^{7}$} \\
\hline Bonds & 0.010 \\
\hline Angles & 1.06 \\
\hline MolProbity Score ${ }^{8}$ & 0.88 \\
\hline Clash Score $^{8}$ & 0.69 \\
\hline Z-Score ${ }^{8}$ (A/B/all) & $0.14 /-0.28 / 0.14$ \\
\hline $\begin{array}{l}\text { RSR of RNA }^{9} \\
(15 \mathrm{~A} / 16 \mathrm{G} / 17 \mathrm{U})\end{array}$ & $0.086 / 0.087 / 0.180$ \\
\hline $\begin{array}{l}\text { RSCC of RNA } \\
(15 \mathrm{~A} / 16 \mathrm{G} / 17 \mathrm{U})\end{array}$ & $0.967 / 0.954$ / 0.926 \\
\hline
\end{tabular}

${ }^{1}$ Statistics for highest resolution bin of reflections in parentheses.

$\left.{ }^{2} R_{\text {sym }}=\Sigma_{h} \Sigma_{j} 1 I_{h j}<I_{h}\right\rangle 1 / \Sigma_{h} \Sigma_{j} I_{h j}$, where $I_{h j}$ is the intensity of observation $j$ of reflection $h$ and $\left\langle\mathrm{I}_{h}\right\rangle$ is the mean intensity for multiply recorded reflections.

${ }^{3}$ Intensity signal-to-noise ratio.

${ }^{4}$ Completeness of the unique diffraction data.

${ }^{5} \mathrm{R}$-factor $=\Sigma_{\mathrm{h}} \mathrm{I} \mathrm{IF}_{\mathrm{o}} \mathrm{I}-\mathrm{IF}_{\mathrm{c}} \mathrm{I} \mathrm{I} / \sum_{\mathrm{h}} \mathrm{IF} \mathrm{I}$, where $\mathrm{F}_{\mathrm{o}}$ and $\mathrm{F}_{\mathrm{c}}$ are the observed and calculated structure factor amplitudes for reflection $\mathrm{h}$.

${ }^{6} \mathrm{R}_{\text {free }}$ is calculated against a $10 \%$ random sampling of the reflections that were removed before structure refinement.

${ }^{7}$ Root mean square deviation of bond lengths and bond angles.

${ }^{8}$ ref. 40

${ }^{9}$ ref. 40 
Table 2. Binding kinetic parameters determined using BLI measurements for UP1 with Wild Type and Cytosine-Substituted SL ${ }^{\mathrm{ESS} 3}$ constructs.

\begin{tabular}{|c|c|c|c|c|}
\hline $\mathrm{SL}^{\mathrm{ESS3}}$ & ${ }^{a}$ Loop Sequence & $\mathrm{k}_{\mathrm{off}}\left(\mathrm{x} 10^{-3} \sec ^{-1}\right)$ & $\mathrm{k}_{\mathrm{on}}\left(\mathrm{x} 10^{5} \mathrm{M}^{-1} \sec ^{-1}\right)$ & $\mathrm{K}_{\mathrm{D}}(\mathrm{nM})$ \\
\hline WT & ...GAUUAG... & $7.5 \pm 0.1$ & $6.8 \pm 1.2$ & $11.8 \pm 2.2$ \\
\hline G8454C & ...CAUUAG... & $8.2 \pm 0.2$ & $1.5 \pm 0.3$ & $58.6 \pm 10.6$ \\
\hline $\mathrm{A} 8455 \mathrm{C}$ & ...GCUUAG... & $9.1 \pm 0.9$ & $3.6 \pm 0.7$ & $27.7 \pm 7.0$ \\
\hline U8456C & ...GACUAG... & $7.8 \pm 0.1$ & $9.1 \pm 1.2$ & $9.0 \pm 1.3$ \\
\hline U8457C & ...GAUCAG... & $7.4 \pm 0.1$ & $2.8 \pm 0.4$ & $27.5 \pm 3.5$ \\
\hline $\mathrm{A} 8458 \mathrm{C}$ & ...GAUUCG... & $8.0 \pm 0.8$ & $0.8 \pm 0.1$ & $112.9 \pm 27.0$ \\
\hline G8459C & ...GAUUAC... & $7.2 \pm 0.1$ & $2.2 \pm 0.1$ & $33.2 \pm 1.0$ \\
\hline
\end{tabular}

${ }^{a}$ Each construct is defined by its mutation in the loop, which is represented in bold in the 5'-to-3' direction. The BLI measurements were performed at $298 \mathrm{~K}$ in $140 \mathrm{mM} \mathrm{K}^{+}$at $\mathrm{pH}$ 6.5. Kinetic parameters are taken as average $+/$ - standard error to the mean of three replicates per construct. 
A.

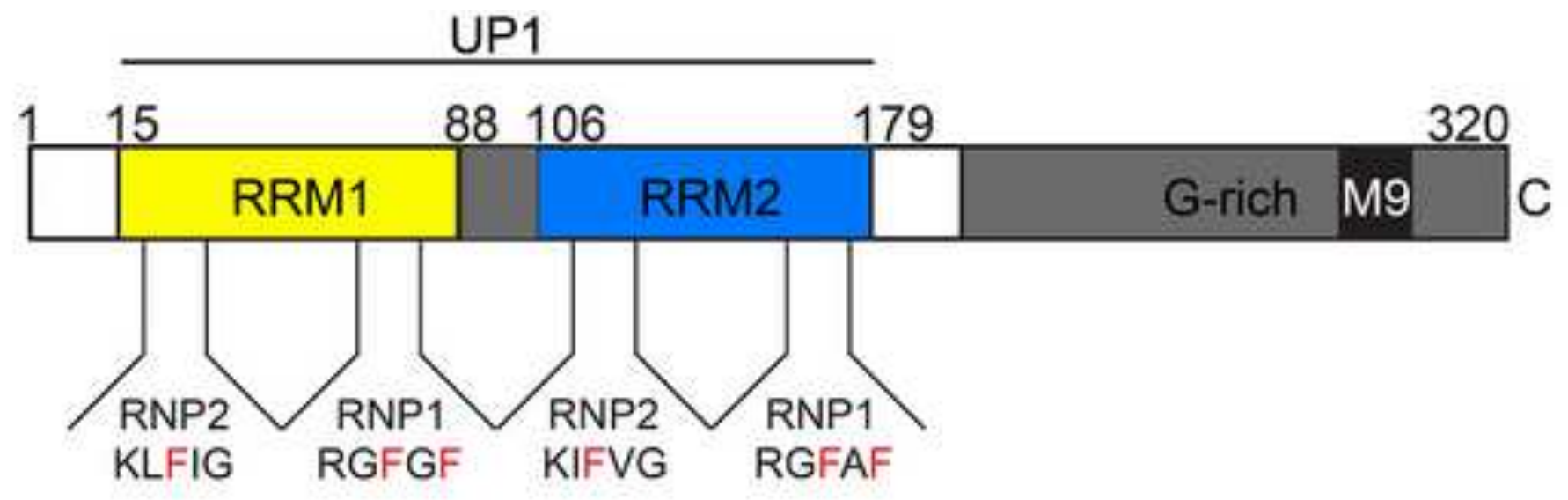

B.

$$
\begin{gathered}
U^{U}{ }^{8458} \\
\text { }{ }^{8455}{ }^{G} \\
C-G \\
U-A 8462 \\
U-A \\
A \quad C \\
C-G \\
C-G \\
U-A 8467 \\
8446 A-U \\
G-C \\
G-C \\
H I V S L 3^{E S S 3}
\end{gathered}
$$

c.

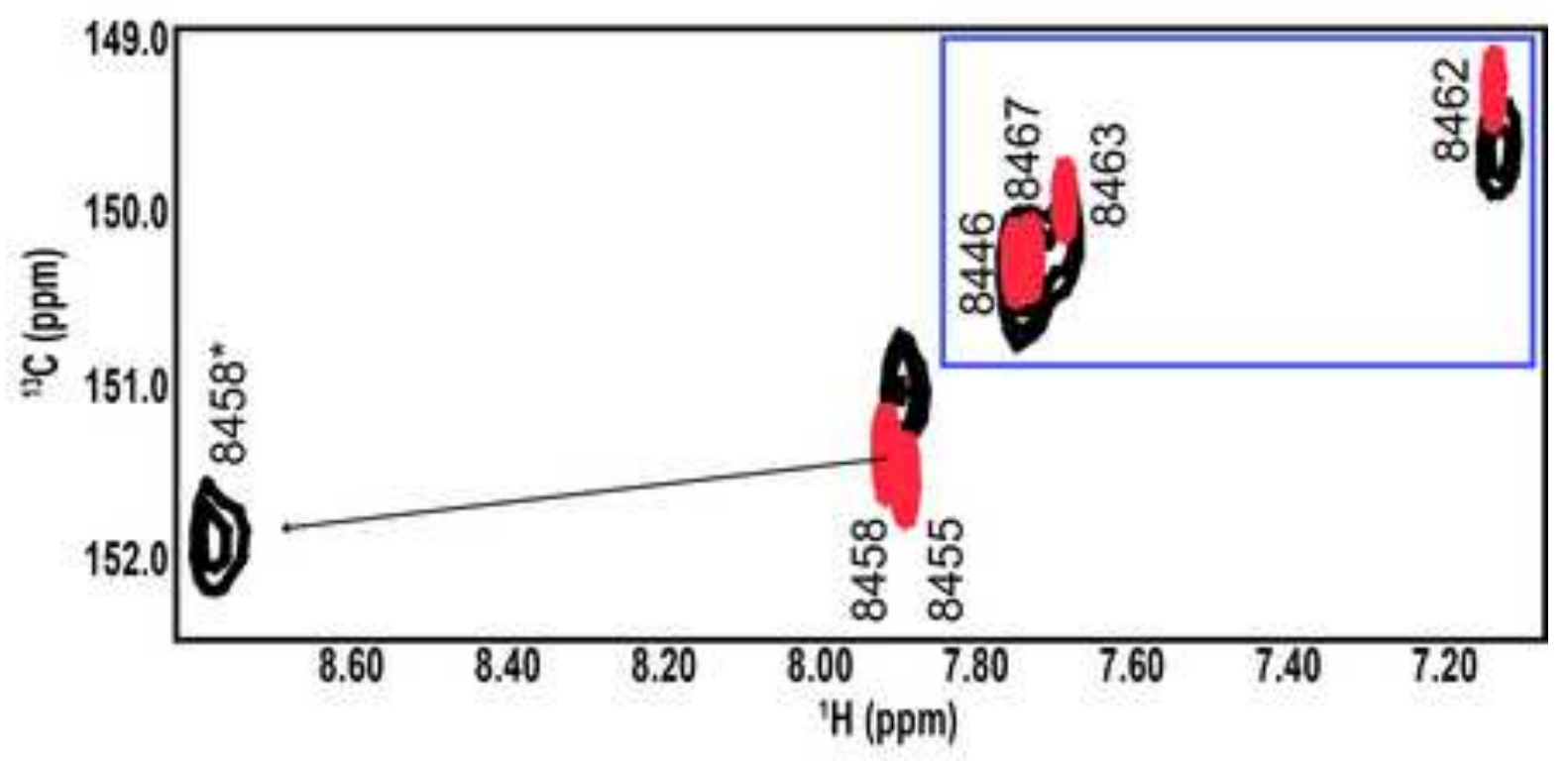


A.

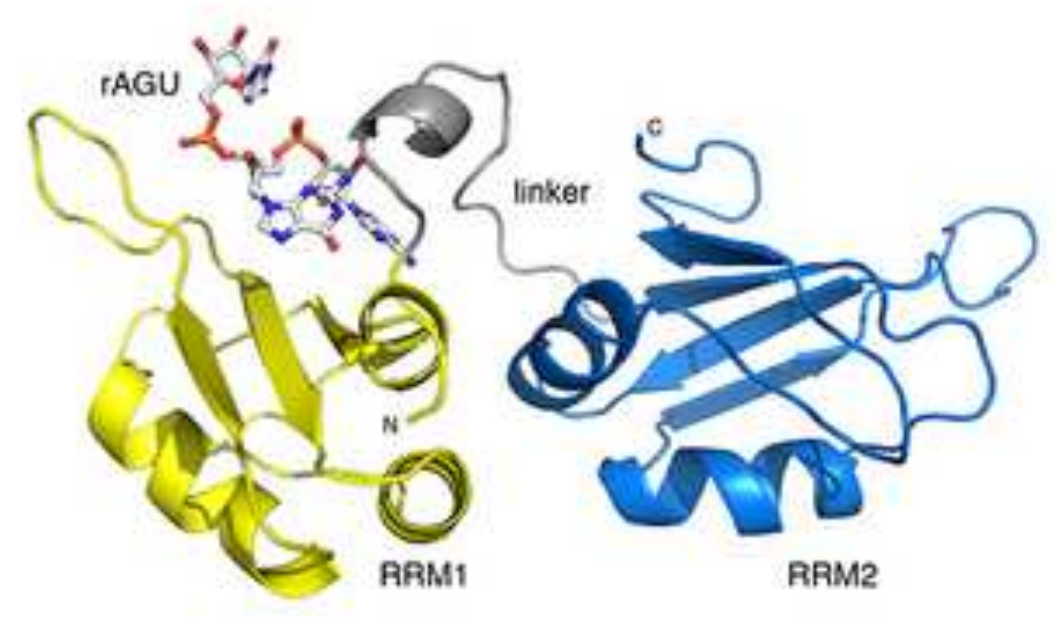

c.

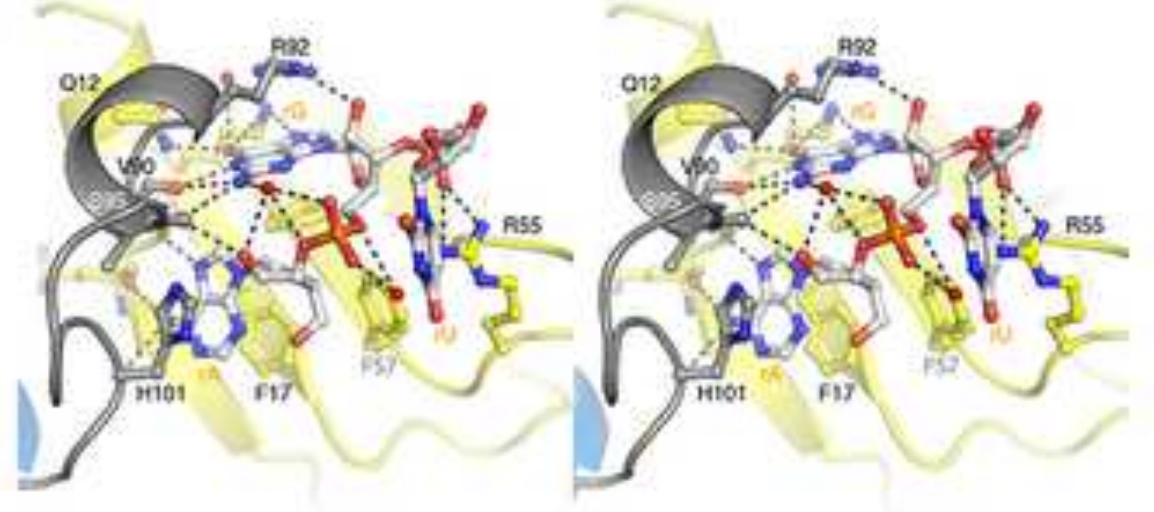

B.

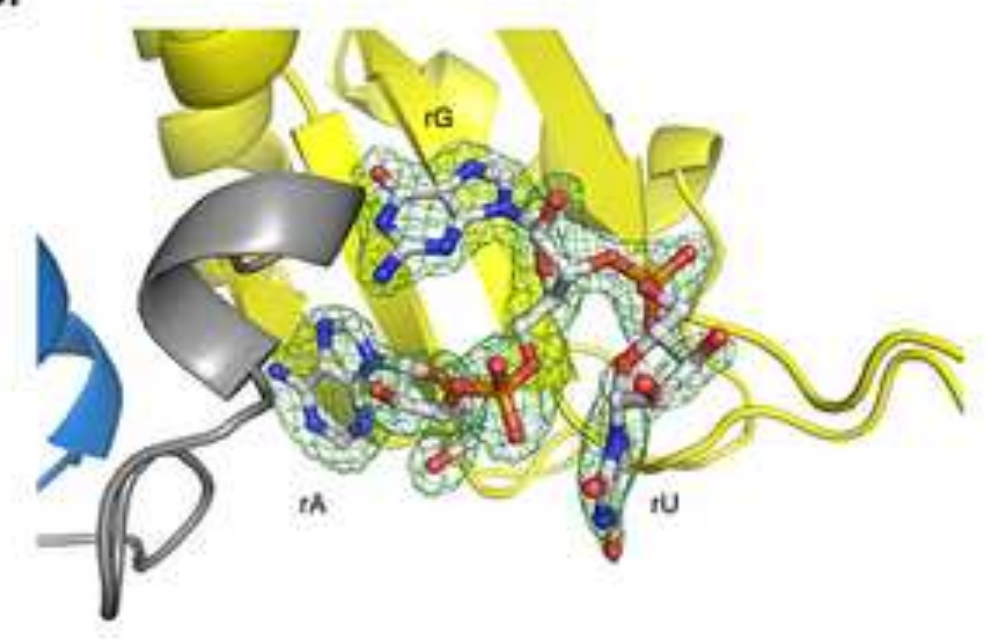

D.

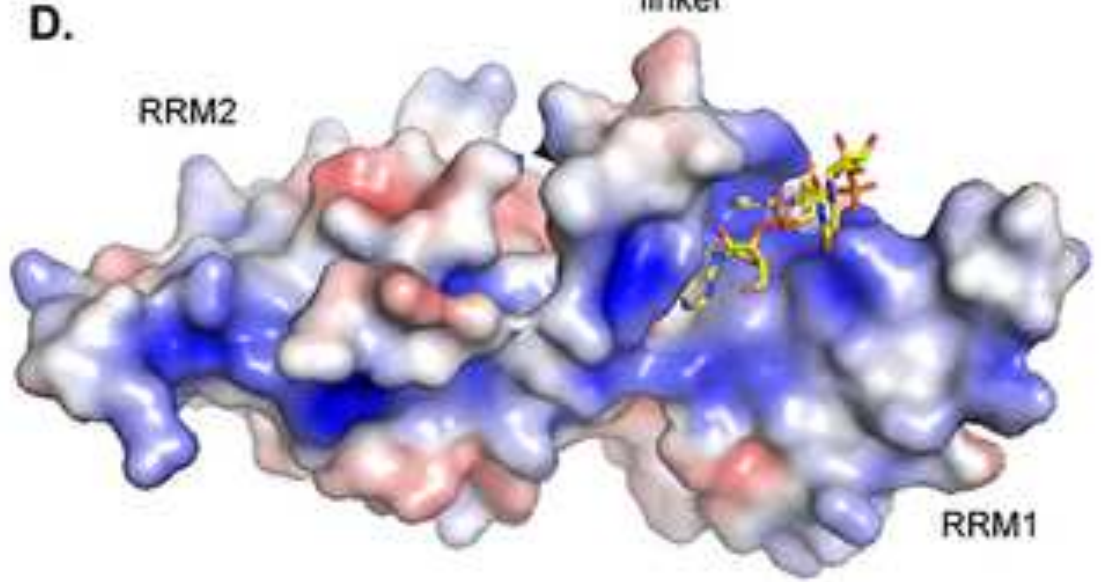


A.
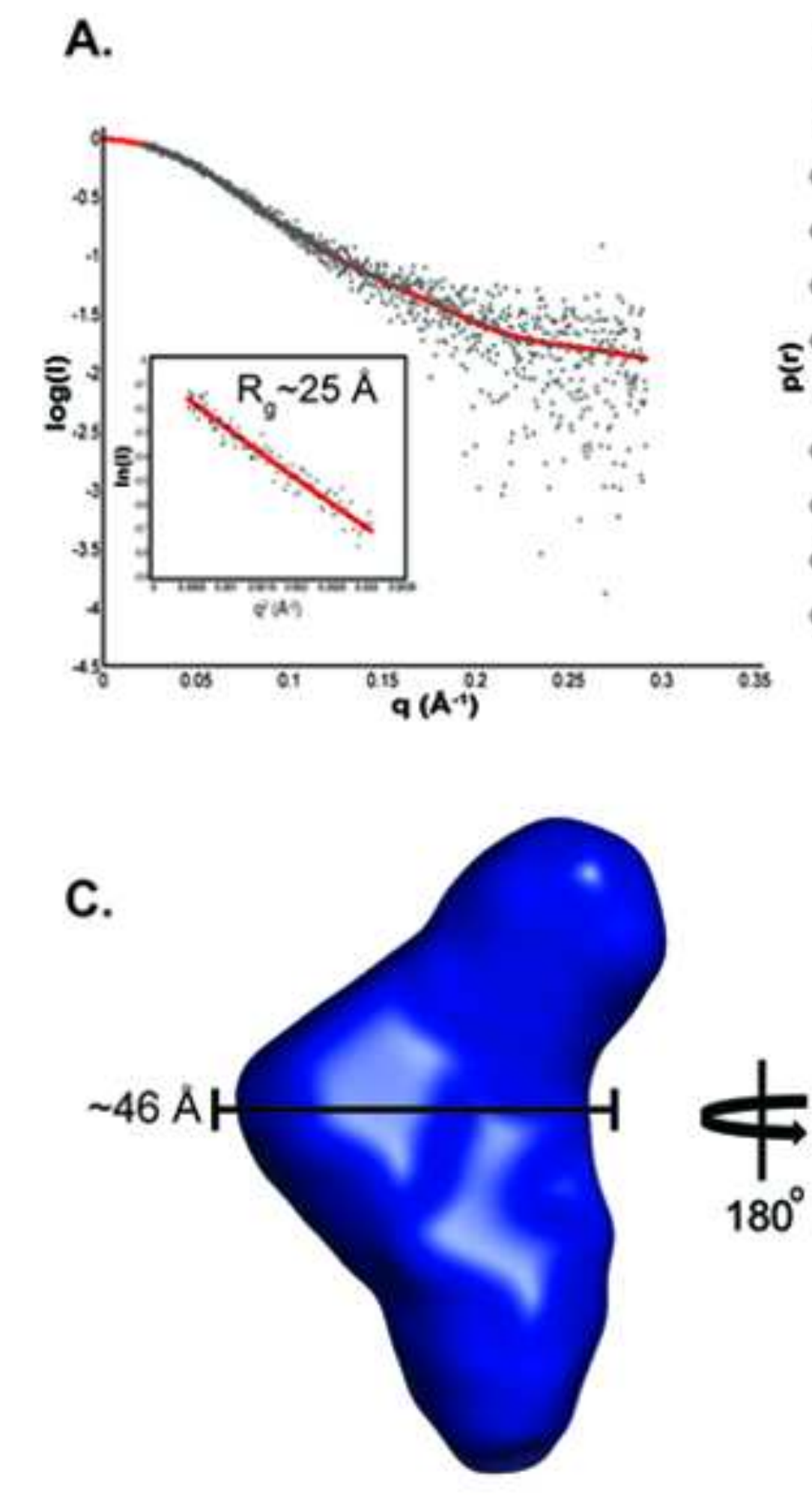

B.

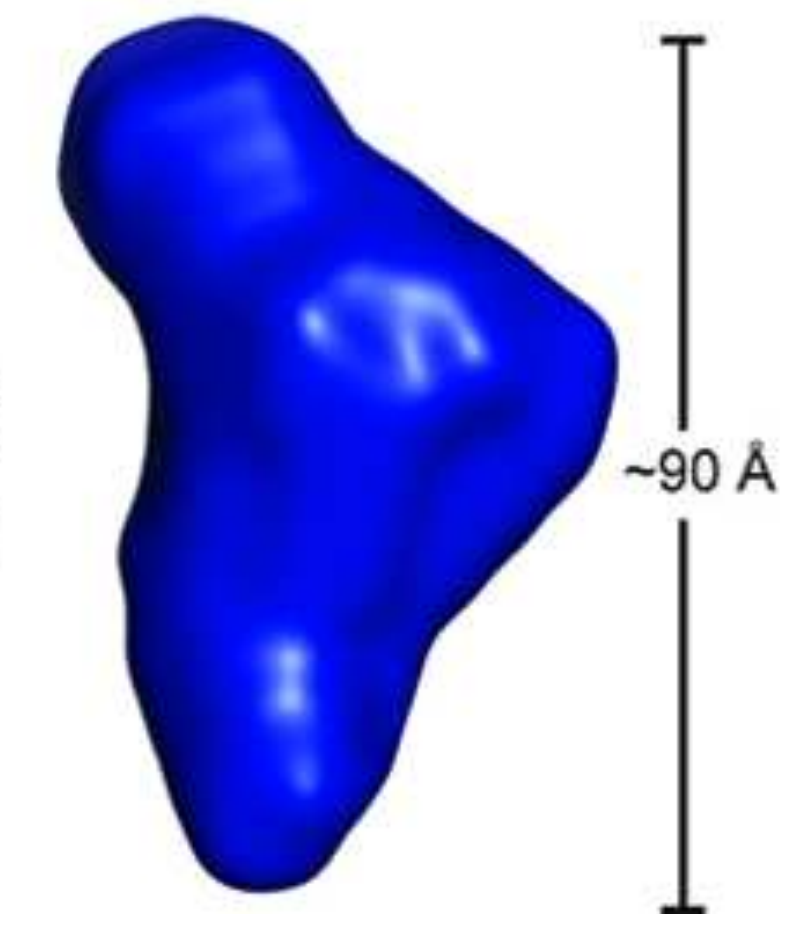

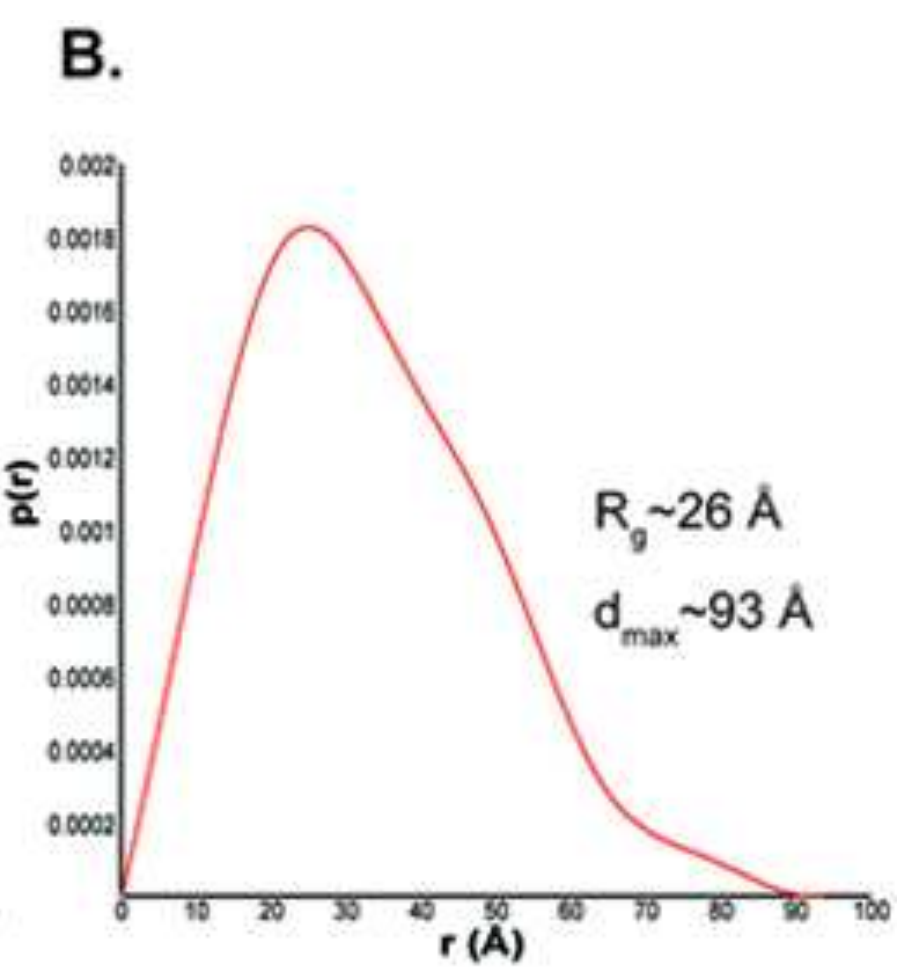

Figure 3
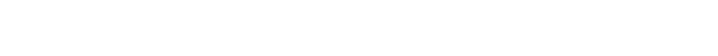
A.

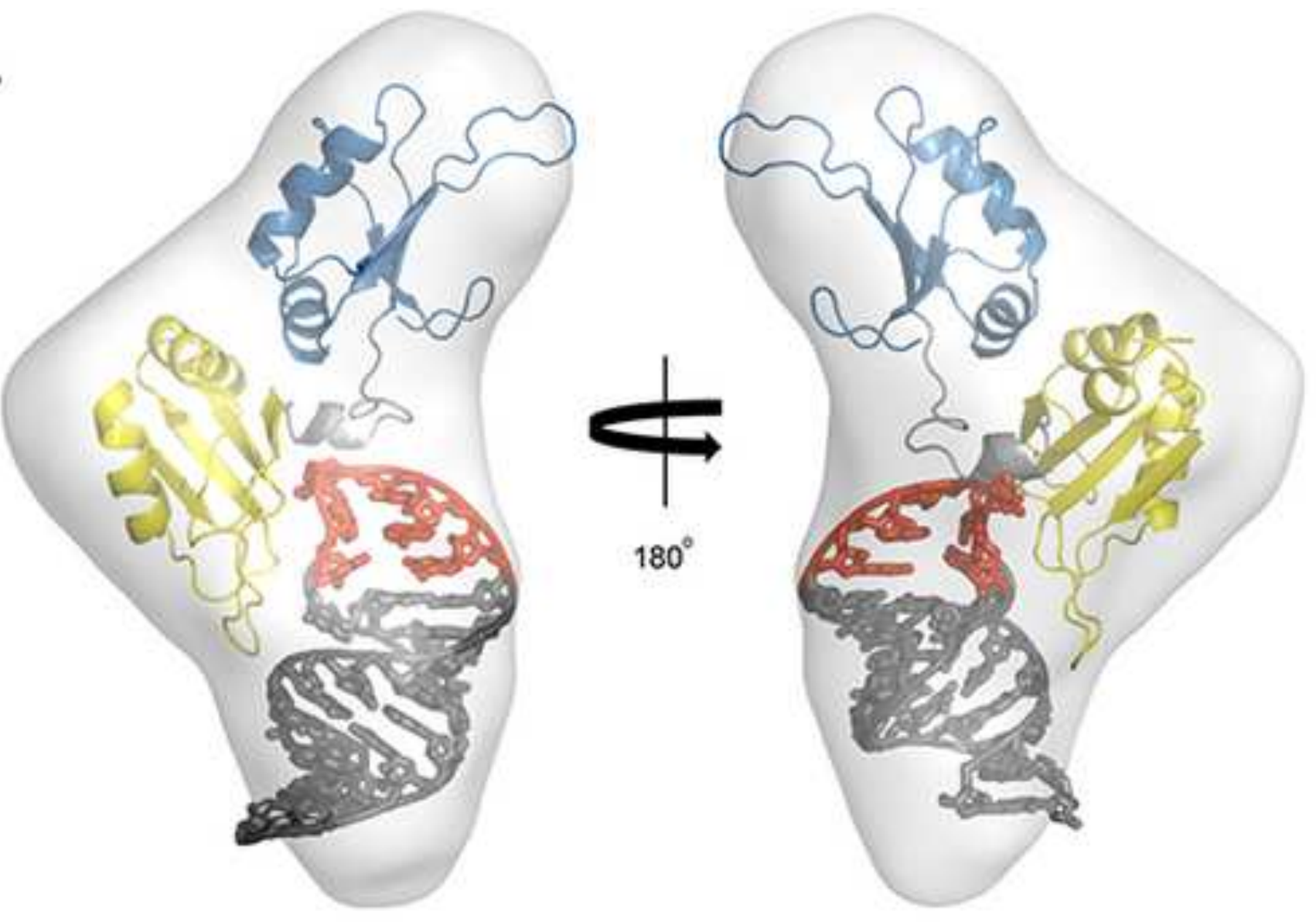

B.

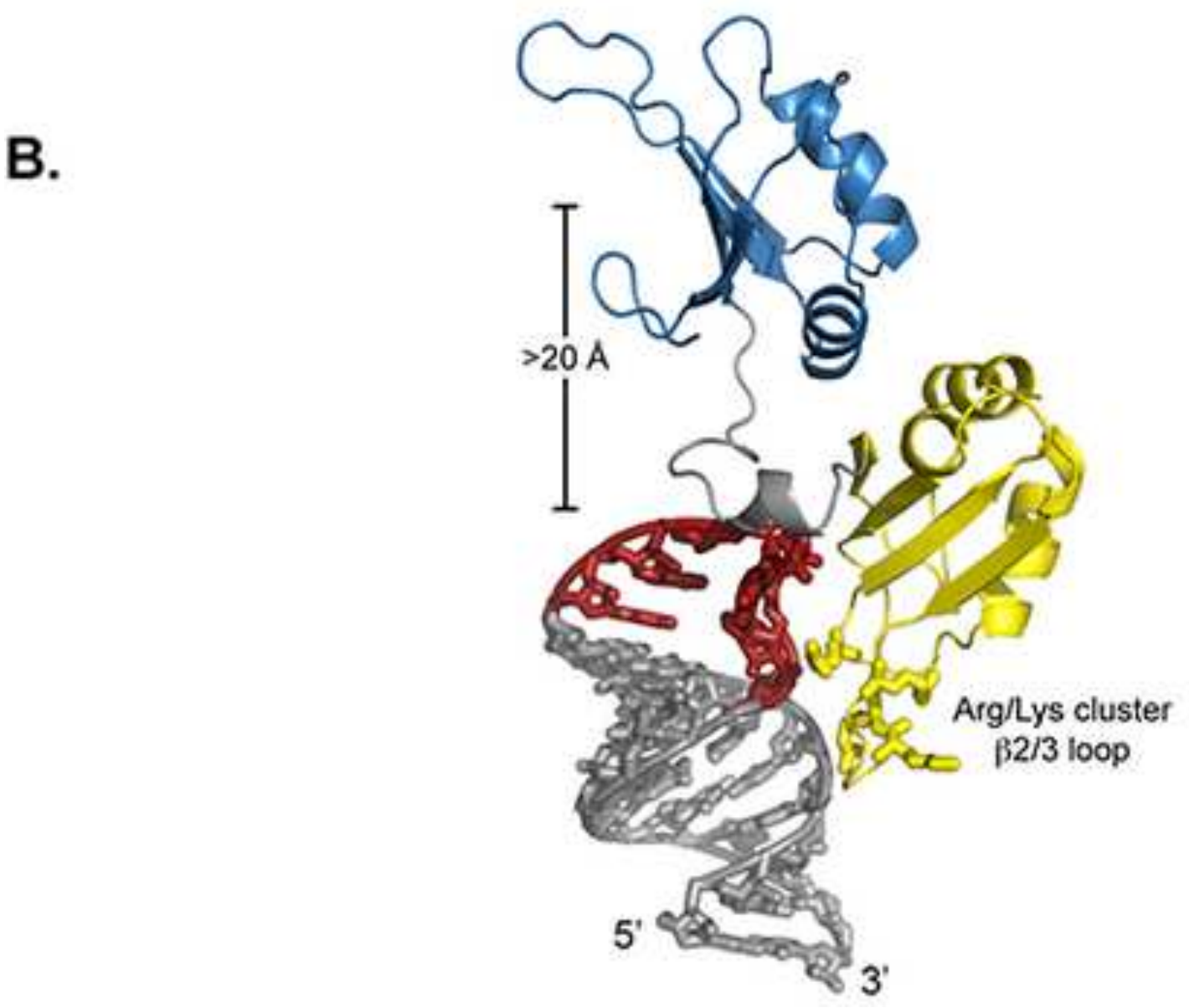

Figure 4 

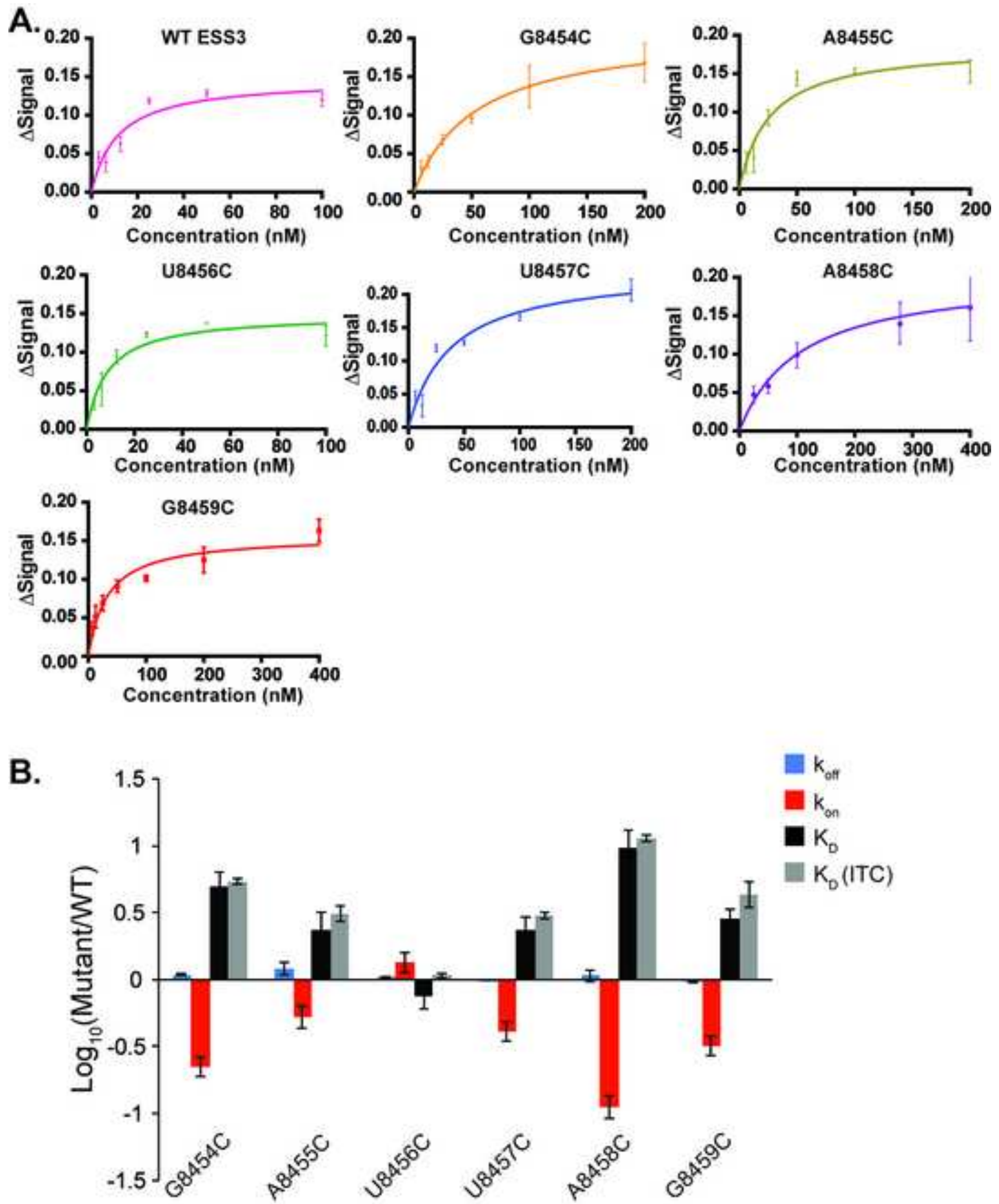
A.

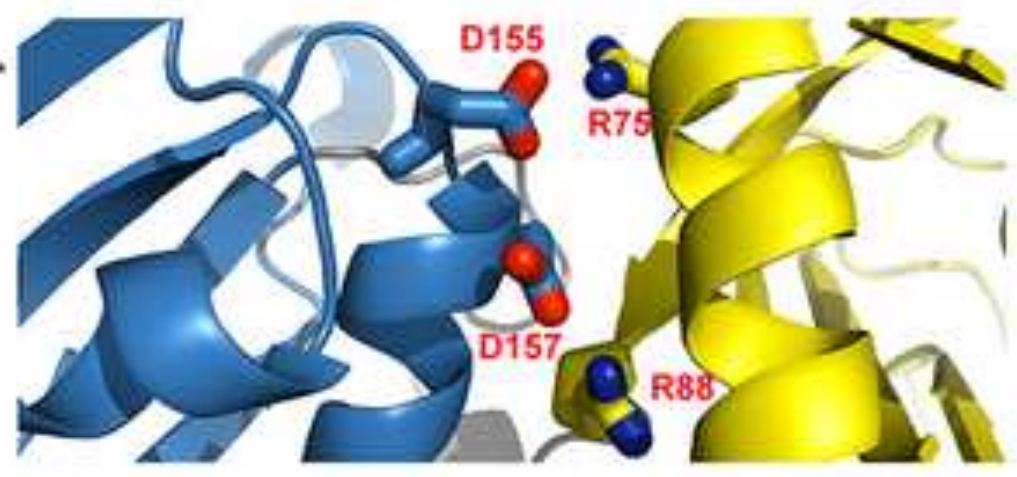

B.

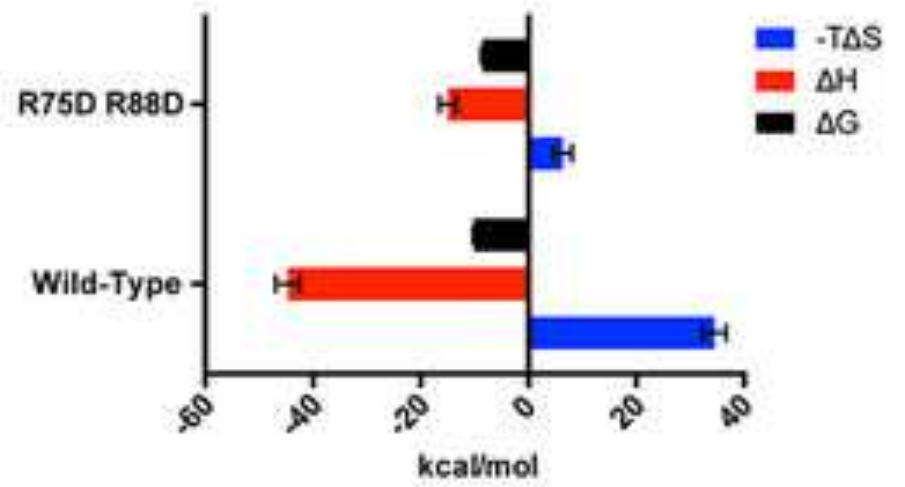

D.

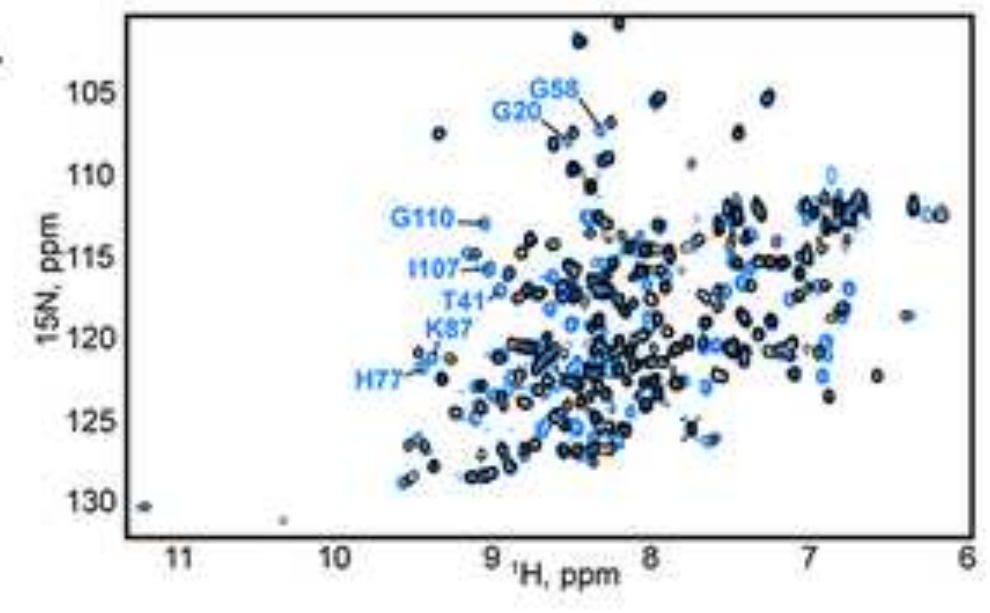

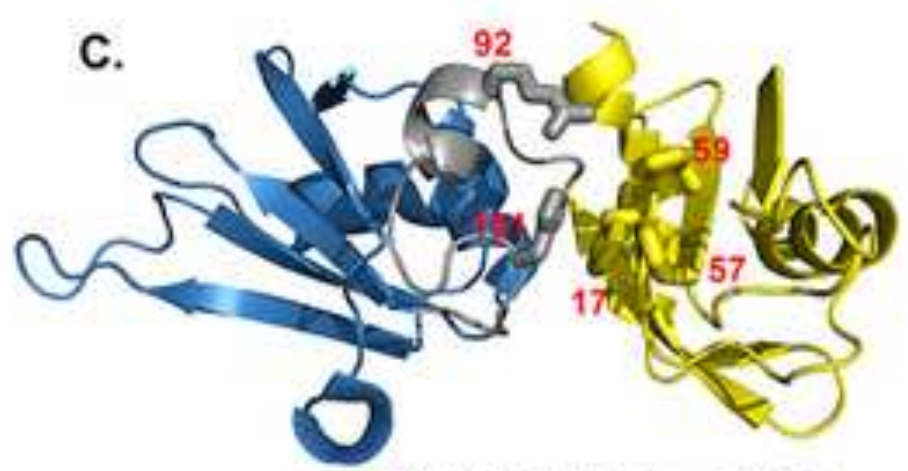

$\mathrm{H}^{104} \cdot \mathrm{F}^{17,57,59}, \mathrm{C} \alpha-\mathrm{C} \alpha \sim 10.2 \mathrm{~A}$

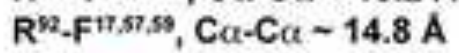

\section{UP1 ${ }^{\mathrm{wT}}, \mathrm{t}=\mathbf{0} \mathrm{ns}$}
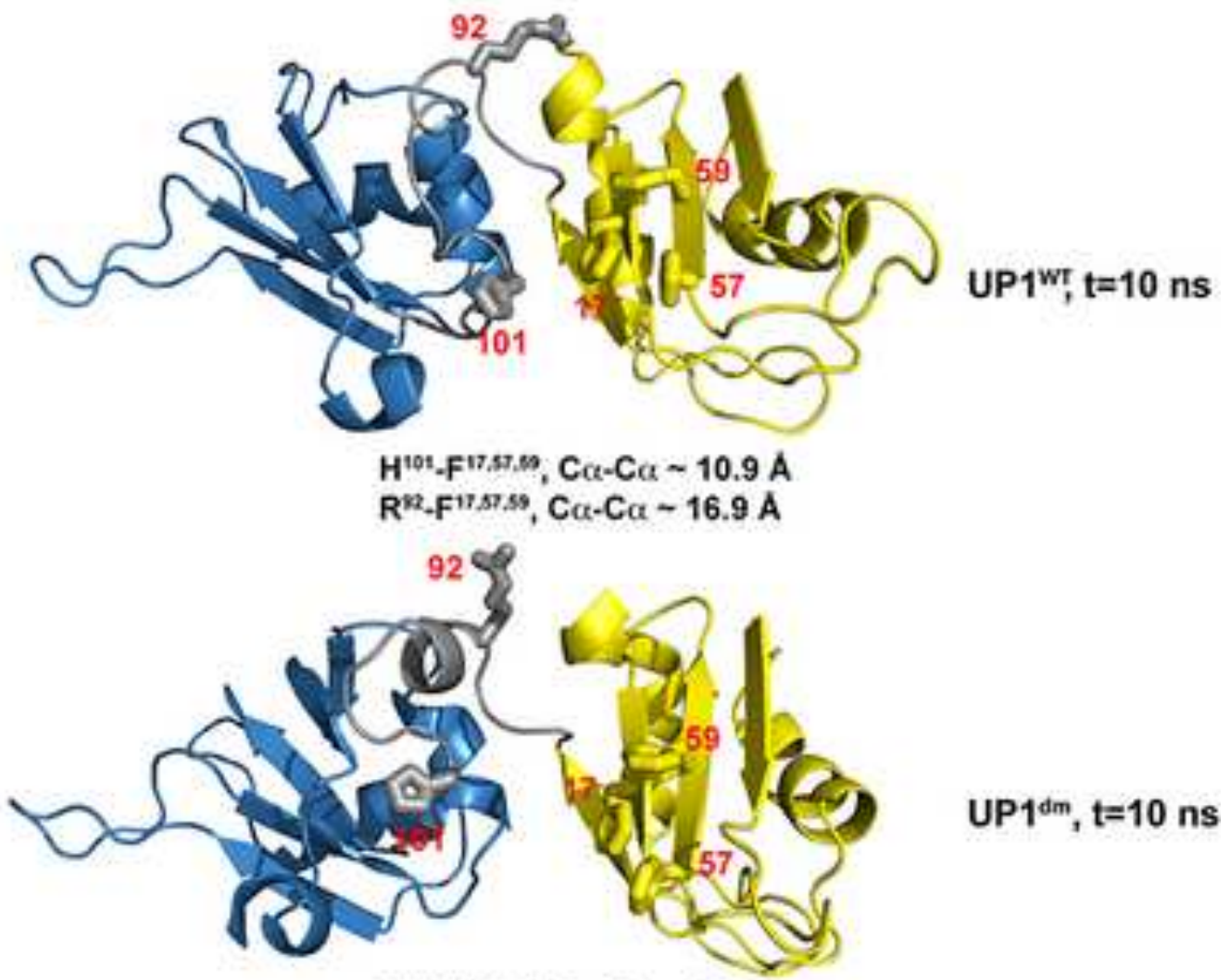

$U P 1^{d m}, t=10 \mathrm{~ns}$

$H^{101}$-Finsts, $\mathrm{C} \alpha-\mathrm{C} \alpha \sim 16.3 \mathrm{~A}$

$\mathrm{R}^{n}$-F $\mathrm{F}^{2.57 s}, \mathrm{C} \alpha-\mathrm{C} \alpha-17.2 \mathrm{~A}$ 
A.
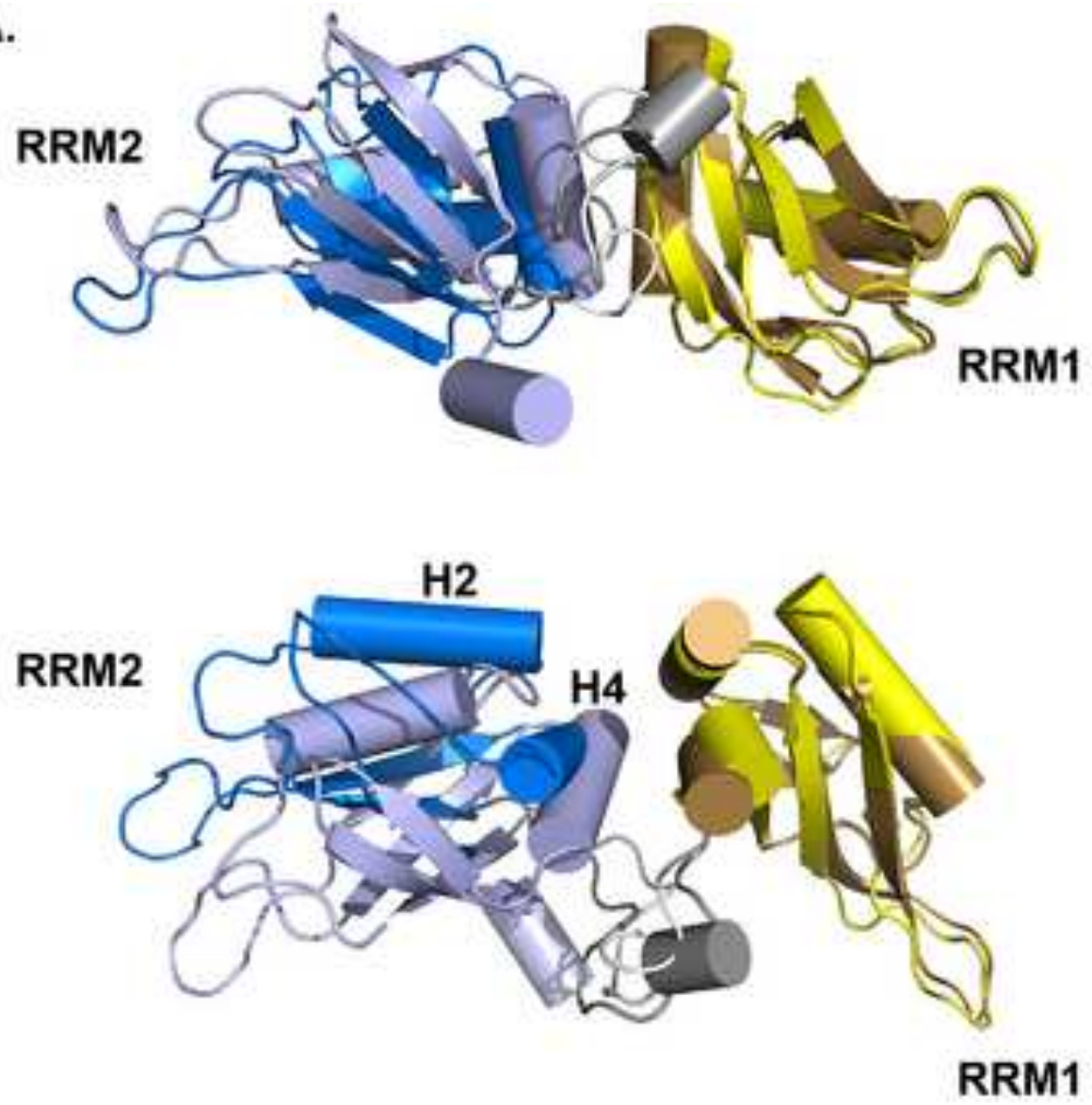

B.
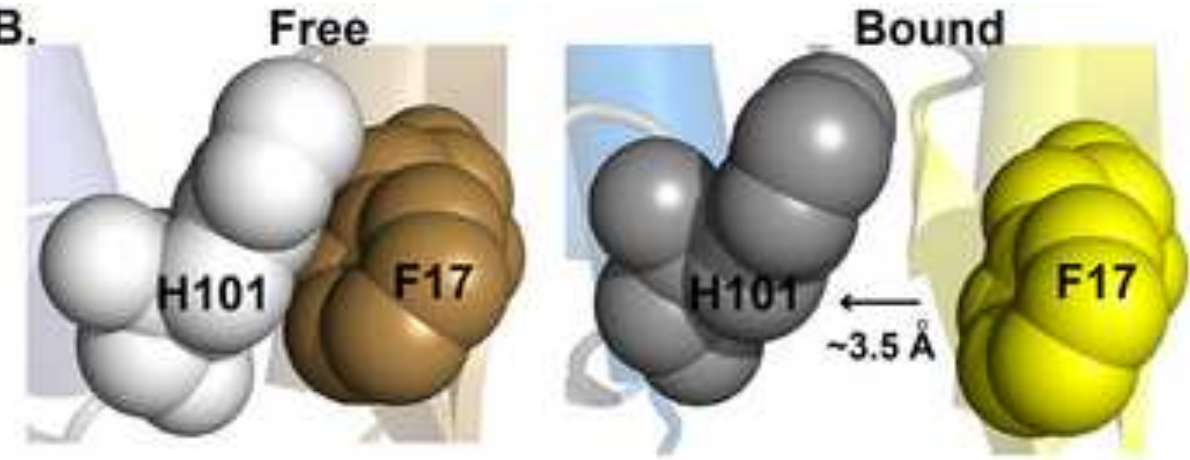

c.

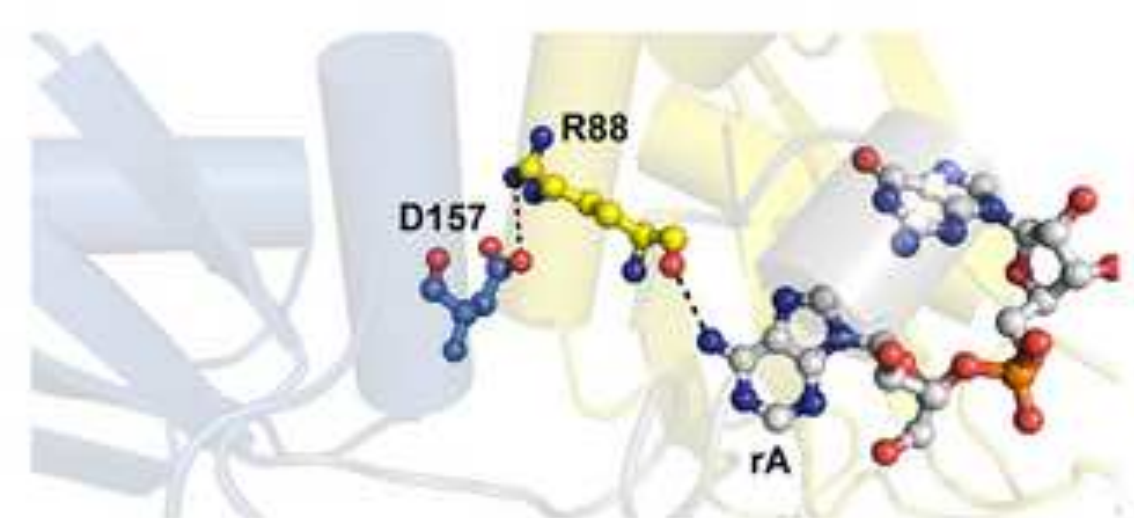

